\title{
Direct fluorescent labeling of NF186 and Nav1.6 in living primary neurons using bioorthogonal click
} chemistry

\author{
Nevena Stajković ${ }^{1,2}$, Yuanyuan Liu ${ }^{3}$, Aleksandra Arsić ${ }^{1,2}$, Ning Meng ${ }^{4}$, Hang Lyu ${ }^{2,3}$, Nan Zhang ${ }^{3}$, Dirk
}

Grimm $^{4,5}$, Holger Lerche ${ }^{3}$, Ivana Nikić-Spiege ${ }^{1^{*}}$

${ }^{1}$ Werner Reichardt Centre for Integrative Neuroscience, University of Tübingen, Tübingen, Germany

${ }^{2}$ Graduate Training Centre of Neuroscience, International Max Planck Research School, University of Tübingen, Tübingen, Germany

${ }^{3}$ Department of Neurology and Epileptology, Hertie Institute for Clinical Brain Research, University of Tübingen, Tübingen, Germany

${ }^{4}$ Virus-Host Interaction Group, Department of Infectious Diseases/Virology, Medical Faculty, University of Heidelberg, Cluster of Excellence CellNetworks, BioQuant, Heidelberg, Germany

${ }^{5}$ German Center for Infection Research and German Center for Cardiovascular Research, partner site Heidelberg, Heidelberg, Germany

*Corresponding author: ivana.nikic@cin.uni-tuebingen.de

\section{Abstract}

The axon initial segment (AIS) is a highly specialized neuronal compartment that regulates the generation of action potentials and maintenance of neuronal polarity. Despite its importance, live imaging of the AIS is challenging due to the limited number of suitable labeling methods. To overcome this limitation, we established a novel approach for live labeling of the AIS using unnatural amino acids (UAAs) and bioorthogonal click chemistry. The small size of the UAAs and the possibility of introducing them virtually anywhere into the target proteins make this method particularly suitable for live labeling and imaging of complex and spatially restricted proteins. With this approach, we labeled two large AIS components, the $186 \mathrm{kDa}$ isoform of neurofascin (NF186) and the $260 \mathrm{kDa}$ voltage-gated sodium channel (Nav1.6), and performed widefield and confocal microscopy in fixed and living neurons. Moreover, we demonstrated the applicability of this method by studying the localization of two epilepsy-causing Nav1.6 variants with a loss-of-function effect. Finally, to further improve the efficiency of the UAA incorporation, we developed adeno-associated viral (AAV) vectors for click labeling in primary neurons. The use of AAV vectors will 
facilitate the transfer of UAA-based click labeling technology to more complex biological systems, such as organotypic slice cultures, organoids, and animal models.

The axon initial segment (AIS) is a highly specialized neuronal compartment responsible for the generation of action potentials (Leterrier, 2018). This unique role of the AIS is mediated by high-density accumulations of voltage-gated ion channels in it (Kole et al., 2008). Particularly important among these channels is Nav1.6, the most abundant voltage-gated sodium channel isoform in the adult mammalian brain (Sole and Tamkun, 2020). Nav1.6 clusters at the distal AIS (Hu et al., 2009) through interactions with the membrane domain of ankyrin G (ankG) (Leterrier, 2018). AnkG acts as an adaptor that anchors Nav1.6 and other important AIS components, such as the $186 \mathrm{kDa}$ neurofascin isoform (NF186), to the underlying cytoskeleton (Leterrier, 2018). As revealed by super-resolution microscopy, AIS constituents are evenly spaced along the AIS with a periodicity of 190 nm (Leterrier et al., 2015, Xu et al., 2013).

Correct function and subcellular targeting of Nav1.6 and NF186 are crucial for proper neuronal activity. Consequently, genetic variations and secondary alterations of Nav1.6 have been implicated in various neurological diseases, such as epilepsy, autism, intellectual disability, movement disorders, and multiple sclerosis (Craner et al., 2004, Johannesen et al., 2021, Meisler et al., 2021), while auto-antibodies against NF186 have been found in multiple sclerosis and chronic inflammatory demyelinating polyradiculoneuropathy (Kira et al., 2019).

To study the trafficking and dynamics of Nav1.6 and NF186, several live-cell labeling approaches have been developed (Akin et al., 2015, Akin et al., 2016, Dzhashiashvili et al., 2007, Gasser et al., 2012, Ghosh et al., 2020, Liu et al., 2021, Sole et al., 2019, Susuki et al., 2013, Zhang et al., 1998). The most widely used approach relies on generating genetic fusions with fluorescent proteins (FPs; Fig. 1a) (Akin et al., 2015, Dzhashiashvili et al., 2007, Gasser et al., 2012, Ghosh et al., 2020, Zhang et al., 1998). The main advantages of FP fusions are their high specificity and compatibility with live-cell imaging. However, most fusions are made by placing the FP at either the $\mathrm{N}$ or $\mathrm{C}$ terminus of the target protein. When it comes to the AIS components, these domains frequently participate in channel inactivation, targeting, and localization, or include binding regions for different regulatory proteins. Some of these interactions may be impaired by 
bioRxiv preprint doi: https://doi.org/10.1101/2022.03.01.480798; this version posted March 3, 2022. The copyright holder for this preprint (which was not certified by peer review) is the author/funder, who has granted bioRxiv a license to display the preprint in perpetuity. It is made available under aCC-BY-NC-ND 4.0 International license.

a

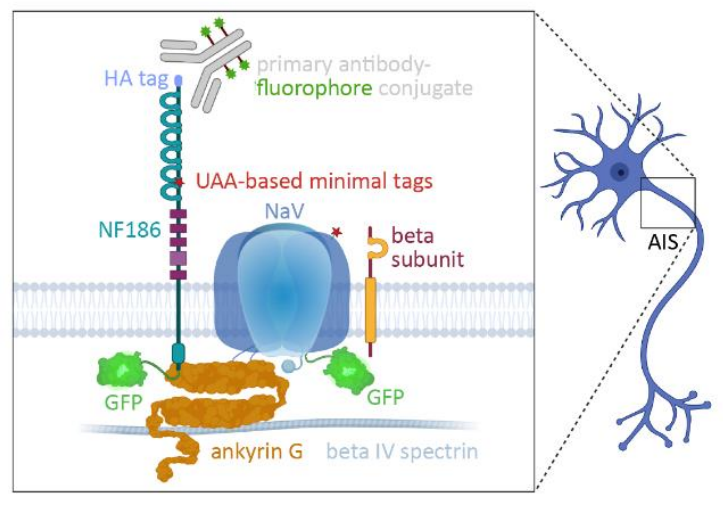

b

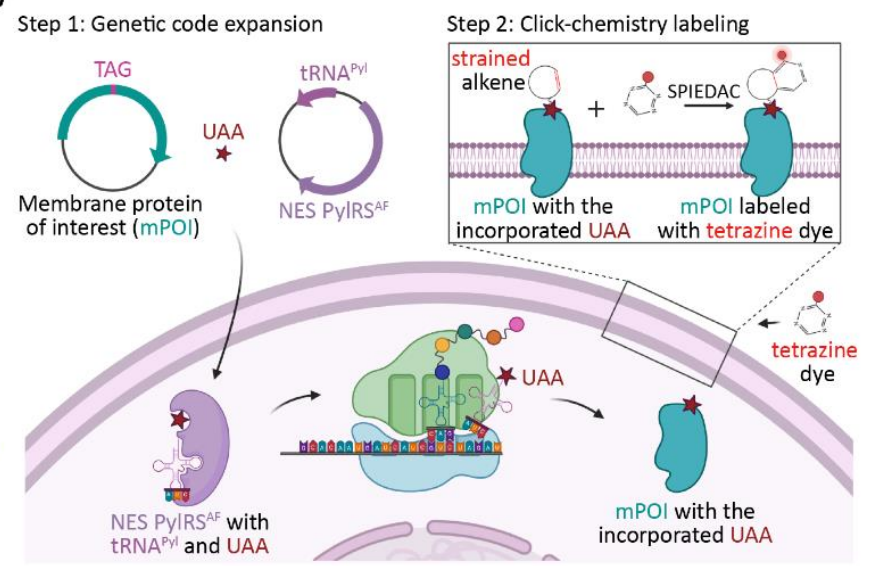

Fig. 1. Schematic overview of the methods for live-cell labeling of the axon initial segment (AIS), including unnatural amino acid (UAA)-based minimal tags. a: Fluorescent protein (FP)-based tags fused to the $C$ terminus are widely used for live labeling of membrane proteins, such as NF186 (Dzhashiashvili et al., 2007) and voltage-gated sodium channels (Akin et al., 2015; Gasser et al., 2012) or submembrane proteins such as ankyrin G (Jenkins et al., 2015). Alternatively, antibodies conjugated to fluorescent dyes can be utilized for live labeling of extracellular domains of membrane proteins, such as NF186 (Evans et al., 2015; Schafer et al., 2009; Torii et al., 2020). As we showed in this study, UAAs and click chemistry can also be used as minimal tags for live-cell labeling of AIS components. b: Schematic representation of genetic code expansion (amber codon suppression) and click labeling of transmembrane proteins. In Step 1, mammalian cells were transfected with plasmids bearing genes that encode a membrane protein of interest [mPOI; that had been mutated to contain an in-frame amber (TAG) stop codon], the Y306A/Y384F (AF) double-mutant of the orthogonal pyrrolysyl (Pyl) tRNA synthetase fused to a nuclear export signal (NES PyIRSAF), and its cognate amber codon suppressor tRNA ${ }^{\text {Pyl }}$ derived from Methanosarcina mazei. During the protein translation, the NES PyIRSAF/tRNA ${ }^{\text {Pyl }}$ pair enabled site-specific incorporation of the UAA, such as trans-cyclooct-2-en-L-lysine (TCO*A-Lys), into the mPOI in response to the TAG stop codon. Subsequently (Step 2), TCO*A-Lys was coupled to a cell-impermeable fluorescent tetrazine dye in a strain-promoted inverse-electron demand Diels-Alder cycloaddition (SPIEDAC) click reaction. The schemes were made in BioRender.com.

fluorescent protein (GFP) to the C terminus of NF186 results in its mislocalization (Dumitrescu et al., 2016,

Dzhashiashvili et al., 2007). In addition to FP fusions, additional methods have been established for live labeling of Nav1.6 and other voltage-gated sodium channel isoforms. One of the approaches for specific labeling of $\mathrm{Nav}$ channels is based on the incorporation of a 17-amino-acid-long biotinylated domain (BAD) into their extracellular domains. The BAD domain, when biotinylated by bacterial biotin ligase, can be labeled with non-permeable streptavidin conjugated dyes. This method has been successfully used to label Nav1.6 (Akin et al., 2015), Nav1.9 (Akin et al., 2019), and Nav1.7 (Sizova et al., 2020) in primary neurons or mammalian cell lines. However, the disadvantages of this approach are the bulkiness and the large size of streptavidin ( $\sim 60 \mathrm{kDa}$ ). Recently, a self-labeling HaloTag fused to the $\mathrm{C}$ termini of endogenous 
endogenous sodium channels, but due to the large size of the HaloTag ( $\sim 33 \mathrm{kDa}$ ), this approach has disadvantages that are similar to those of FPs.

Alternatively to genetic fusions, immunostaining with fluorescent antibodies can be used for AIS labeling in living neurons. For this purpose, antibodies that recognize either extracellular domains of endogenous AIS proteins or short tags attached to the extracellular domains of recombinant/endogenous AIS components have been utilized (Fig. 1a) (Dumitrescu et al., 2016, Dzhashiashvili et al., 2007, Evans et al., 2015, Freal et al., 2019, Hedstrom et al., 2008, Liu et al., 2021, Schafer et al., 2009, Torii et al., 2020). However, antibodies, due to their multivalence, can induce crosslinking, which makes them unsuitable to study the dynamics of the AIS, such as plasticity (Dumitrescu et al., 2016). Furthermore, when it comes to the labeling of closely-related proteins such as different $\mathrm{Nav}$ isoforms, antibodies do not always provide enough specificity. Keeping these limitations in mind, it would be beneficial to develop additional approaches for direct and minimally invasive live-cell labeling of AIS components.

We and other researchers previously developed unnatural amino acid (UAA)-based minimal tags for live-cell protein labeling in mammalian cells (Lang et al., 2012b, Lang et al., 2012a, Nikic et al., 2014, Plass et al., 2012, Uttamapinant et al., 2015). These tags are based on site-specific labeling of a single UAA with a fluorescent dye. With the help of genetic code expansion (Chin, 2017, Nikic-Spiegel, 2020, Wang, 2017), UAAs carrying strained alkenes are co-translationally incorporated into the protein of interest in response to an in-frame amber stop codon (Fig. 1b). Subsequently, fluorescent dyes are directly attached to the UAAs with click chemistry reactions. One such reaction is the bioorthogonal catalyst-free strain-promoted inverse-electron demand Diels-Alder cycloaddition (SPIEDAC) between UAAs that contain strained alkenes and tetrazine derivatives of fluorescent dyes. Due to its high reaction rates and biorthogonality, SPIEDAC is particularly useful for live-cell labeling. In recent years, this type of labeling has emerged as one of the most powerful approaches for the minimally invasive labeling of both extracellular and intracellular proteins in standard cell lines. We have recently established this type of labeling in primary neurons by labeling the neuronal cytoskeleton (Arsić et al., 2022). In parallel, this approach has been used to label small (34-45 kDa) transmembrane AMPA receptor regulatory proteins (Bessa-Neto et al., 2021).

In this study, we utilized SPIEDAC to label two large transmembrane AIS proteins: NF186 ( $186 \mathrm{kDa})$ and Nav1.6 ( 260 kDa). Direct fluorescent labeling of the UAA with small dyes allowed us to perform fixed and live-cell confocal microscopy and to study localization of WT and pathogenic Nav1.6 variants. We also developed adeno-associated viral (AAV) vectors that mediated high-efficiency delivery of the genetic code expansion components to primary neurons. 
100

101

102

103

104

105

106

107

108

109

110

111

112

113

114

115

116

117

118

119

120

121

122

123

124

125

126

127

\section{Results}

\section{Genetic code expansion and click labeling of NF186 in the ND7/23 cell line}

To establish live-cell labeling of the AIS using click chemistry, we first focused on the labeling of one of its smaller (186 kDa) components, NF186 (Fig. 1a). Before introducing amber codon (TAG) labeling sites via site-directed mutagenesis, we modified the previously described plasmid (Zhang et al., 1998) by moving the hemagglutinin (HA) tag from the $\mathrm{N}$ terminus to the $\mathrm{C}$ terminus. Since this plasmid encodes the wild-type (WT) rat NF186 driven by a cytomegalovirus (CMV) promoter, we will refer to it hereafter as a "CMV-NF186 ${ }^{\mathrm{WT}}$-HA" construct. The C-terminal HA-tag allowed us to detect the full-length NF186 protein by immunostaining it with an anti-HA antibody. To find the optimal TAG mutant in terms of expression and click labeling efficiency, we tested different TAG positions in NF186. Testing different positions for click labeling of a target protein is an important step, considering that the amber codon suppression efficiency depends on the surrounding sequence (Bartoschek et al., 2021), while the labeling efficiency depends on the accessibility of a UAA to a tetrazine dye. SWISS-MODEL (Bienert et al., 2017), based on the crystal structure of a titin fragment (PDB 3B43) (von Castelmur et al., 2008) was used to select six potential positions for the UAA incorporation in the extracellular domain of NF186 (Fig. 2a). Then, we introduced the corresponding amber (TAG) codons into the rat Nfasc gene by site-specifically mutating

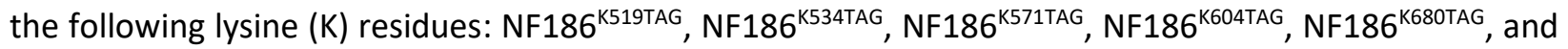
NF186 ${ }^{\mathrm{K} 809 T A G}$.

We have recently shown that the rodent neuroblastoma ND7/23 cell line is a suitable host for genetic code expansion and click labeling of neuronal proteins (Arsić et al., 2022). To identify the optimal TAG position for NF186 click labeling, we co-transfected ND7/23 cells with CMV-NF186 ${ }^{\text {TAG }}$-HA constructs and the previously described codon-optimized plasmid for clickable UAA incorporation. The latter encodes the Y306A/Y384F (AF) double mutant of the Methanosarcina mazei-derived orthogonal pyrrolysyl (Pyl) tRNA synthetase fused to a nuclear export signal (NES PyIRS ${ }^{\mathrm{AF}}$ ) and its cognate amber codon suppressor tRNA ${ }^{\mathrm{Pyl}}$ (Arsić et al., 2022). The immunostaining with an anti-HA antibody and confocal imaging confirmed that all the mutants were expressed in the presence of the trans-cyclooct-2-en-L-lysine (TCO*A-Lys) UAA, whereas no expression was detected in the absence of TCO*A-Lys (Supplementary Fig. 1 and 2). Furthermore, live-cell click labeling with an ATTO488-tetrazine derivative (ATTO488-tz) showed that all six 
bioRxiv preprint doi: https://doi.org/10.1101/2022.03.01.480798; this version posted March 3, 2022. The copyright holder for this preprint (which was not certified by peer review) is the author/funder, who has granted bioRxiv a license to display the preprint in perpetuity. It is made available under aCC-BY-NC-ND 4.0 International license. in the absence of UAA, there was no click labeling (Supplementary Fig. 2).

a
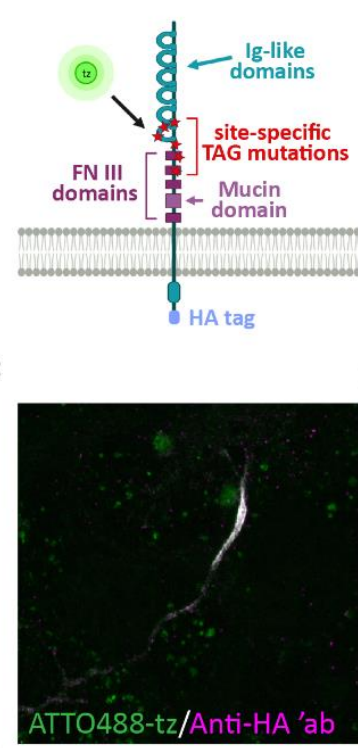

d

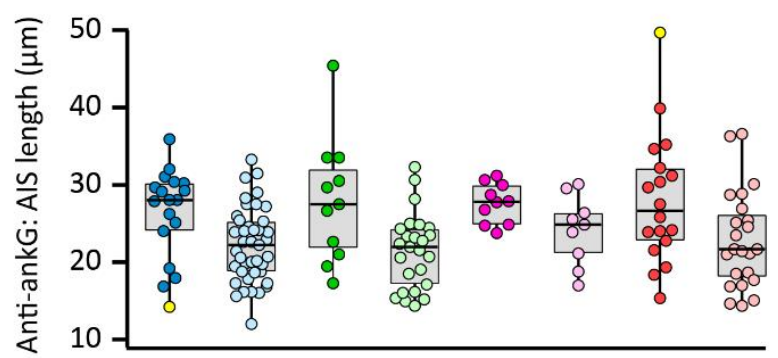

b

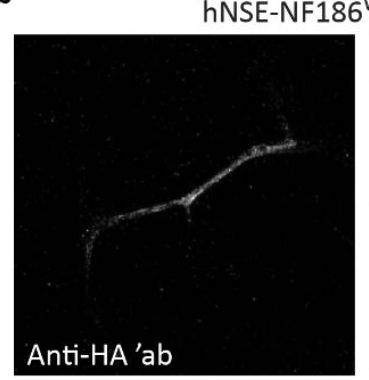

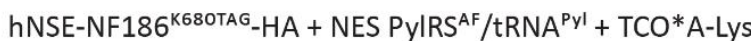

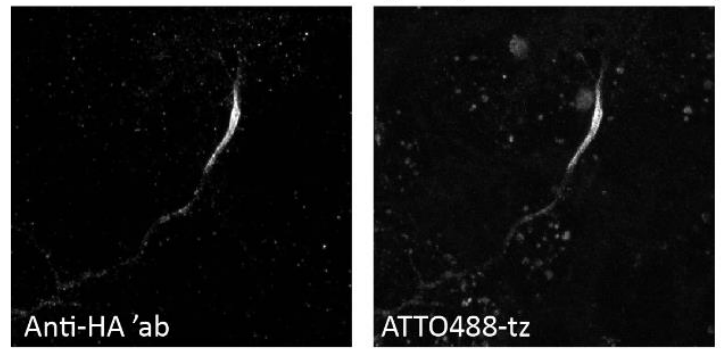

- WT HA+

○ WT HA-

- K519TAG HA+

- K519TAG HA-

- K604TAG HA+

- K604TAG HA-

- K680TAG HA+

- K680TAG HA-

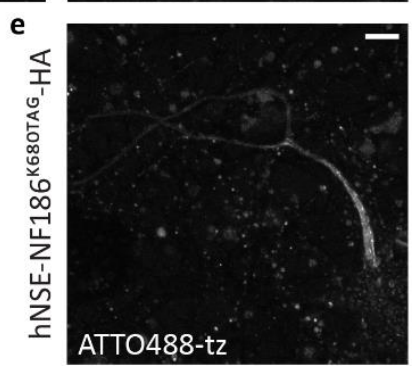

Fig. 2. Genetic code expansion and click labeling of NF186 in living primary neurons. a: Schematic representation of the NF186 structure with clickable labeling sites (red) and the C-terminal hemagglutinin (HA) tag (purple). b-c: Representative confocal images of primary rat cortical neurons at day in vitro (DIV) 11 expressing NES PyIRSAF/tRNA ${ }^{\text {Pyl }}$ and NF186WT-HA or

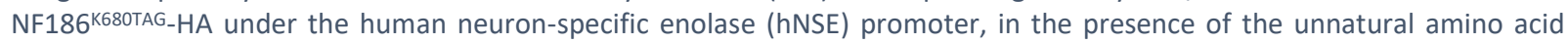
TCO*A-Lys. Prior to imaging, living neurons were click-labeled with ATTO488-tetrazine (tz), fixed, and immunostained with anti-HA and anti-ankyrin G (ankG) primary antibodies, followed by Alexa Fluor (AF) 555- and AF633-conjugated secondary antibodies. $\mathbf{d}$ : Distribution of the AIS lengths measured in confocal images of the anti-ankG immunostained rat neurons

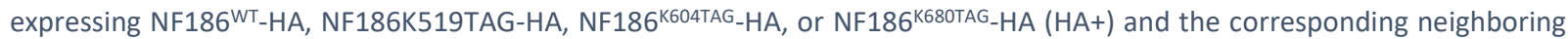
untransfected (HA-) neurons. The box plots indicate the median (the black lines inside the box), the $25^{\text {th }}$ and $75^{\text {th }}$ percentiles (the box boundaries), the single data points (the dots), and the outliers (the yellow dots). The whiskers lengths are defined by the minimum and maximum data points. The non-parametric Kruskal-Wallis test with Dunn-Bonferroni posthoc did not show any significant differences between the HA+ and HA- AIS lengths for WT and the three clickable mutants $\left(p_{W T}=0.206\right.$, $p_{\text {K519TAG }}=0.265 p_{\text {K604TAG }}=1.000$, and $p_{\text {K680TAG }}=0.605$; number $(n)$ of analyzed cells: $n_{W T H A+}=18, n_{W T ~ H A-}=41, n_{K 519 T A G ~ H A+}=11$, $n_{\text {K519TAG HA- }}=25, n_{\text {K604TAG HA+ }}=10, n_{\text {K604TAG HA- }}=9, n_{\text {K680TAG HA+ }}=18$, and $\left.n_{\text {K680TAG HA- }}=23\right)$. The details of the statistical analysis are shown in Supplementary Table 1. e: Representative confocal image of a living neuron at DIV 11 expressing NF186 ${ }^{\text {K680TAG-HA }}$ labeled with ATTO488-tz. The Z-stack images are shown as maximum intensity projections in all panels. Scale bars: $10 \mu \mathrm{m}$ (b) c, e). The scheme in panel a was made in BioRender.com.

\section{Genetic code expansion and click labeling of NF186 in living primary neurons}

Having established click labeling of NF186 ${ }^{\mathrm{TAG}}-\mathrm{HA}$ in the ND7/23 cell line, we aimed to implement 132 labeling of NF186 in primary rat cortical neurons. Typically, we would pick the best expressing TAG mutant 
with the highest labeling efficiency from the ND7/23 screen, since this construct has the highest chance of being successfully expressed and labeled in primary neurons. However, it is well established that overexpression of certain AIS components such as ankG and NF186 can lead to their mislocalization or can result in an abnormally elongated AIS (Dumitrescu et al., 2016, Galiano et al., 2012, Hamdan et al., 2020, Jenkins et al., 2015). Considering that the NF186 ${ }^{\text {TAG }}-H A$ mutants from our ND7/23 screen had a lower expression than the NF186 ${ }^{\mathrm{WT}}-\mathrm{HA}$, we anticipated that this would overcome mislocalization problem. For confirmation, we tested all six NF186 ${ }^{\mathrm{TAG}}-\mathrm{HA}$ mutants in the primary neurons (Supplementary Fig. 3). Four days after the transfection, on day in vitro (DIV) 11, we performed immunostaining with an anti-HA antibody to estimate the expression level and localization of the recombinant NF186. As expected, the NF186 ${ }^{\mathrm{WT}}$-HA expressed from a strong CMV promoter was mostly mislocalized and expressed in the soma and other neuronal processes (Supplementary Fig. 3a). NF186 ${ }^{\mathrm{TAG}}-\mathrm{HA}$ mutants, despite their lower expression levels, also frequently showed ectopic localization along distal axons (Supplementary Fig. 3b).

To improve the localization of the NF186-HA constructs, we replaced the CMV promoter with the weaker human neuron-specific enolase promoter (hNSE) in the NF186 ${ }^{\mathrm{WT}}$-HA and NF186 ${ }^{\mathrm{TAG}}$-HA constructs (hereafter referred to as "hNSE-NF186 ${ }^{\mathrm{WT}}-\mathrm{HA}$ " and "hNSE-NF186 ${ }^{\mathrm{TAG}}-\mathrm{HA}$ "). This promoter was previously used to lower the expression level of NF186 in hippocampal neurons (Hamdan et al., 2020). Four days after the transfections, we performed click labeling with ATTO488-tz, followed by immunostaining with anti-HA and anti-ankG antibodies (Fig. 2b-c, Supplementary Fig. 4). Confocal microscopy revealed that the hNSE promoter lowered the WT and clickable NF186-HA expression levels and consequently improved the localization of these proteins (Fig. 2b-c and Supplementary Fig. 4). Furthermore, confocal microscopy confirmed that only the hNSE-NF186 ${ }^{\text {TAG }}$-HA constructs and not hNSE-NF186 ${ }^{\text {WT }}$-HA could be labeled with click chemistry. Finally, these experiments revealed that not all the NF186 ${ }^{\text {TAG }}$ mutants could be equally well labeled with ATTO488-tz in primary neurons (Fig. 2c and Supplementary Fig. 4). For example, the number of transfected neurons with NF186 ${ }^{\mathrm{K} 534 \mathrm{TAG}}-\mathrm{HA}$ and NF186 ${ }^{\mathrm{K} 571 \mathrm{TAG}}-\mathrm{HA}$ constructs was lower, and click labeling was either weak or completely absent (Supplementary Fig. 4). Therefore, we excluded these two mutants from our further analysis. Although NF186 ${ }^{\mathrm{K} 809 \mathrm{TAG}}$-HA (Supplementary Fig. 4) showed bright click labeling, we excluded it from the analysis due to its frequent ectopic expression along the distal axon.

To identify the most suitable TAG position for NF186 click labeling among the three remaining mutants (NF186 ${ }^{\text {K519TAG }}-\mathrm{HA}, \mathrm{NF186}{ }^{\mathrm{K} 604 \mathrm{TAG}}-\mathrm{HA}$, and NF186 ${ }^{\mathrm{K} 680 \mathrm{TAG}}-\mathrm{HA}$ ), we assessed whether the AIS structure was affected by overexpression of the hNSE-NF186 ${ }^{\text {TAG }}$-HA constructs. To this end, we used the previously custom-written MATLAB script for quantitative measurements of the AIS length (Grubb, 2021, Grubb and 
Burrone, 2010) to compare the AIS length of the NF186 ${ }^{\mathrm{WT} / \mathrm{TAG}}-\mathrm{HA}$ transfected (HA positive) and neighboring untransfected (HA negative) neurons (Fig. 2d). Anti-HA immunostaining allowed us to identify transfected neurons, while we used immunostaining with anti-ankG as a transfection-independent marker to identify the AIS in both transfected and surrounding untransfected neurons. Quantitative analysis of the AIS length showed no significant differences between the AIS length of the neurons that expressed recombinant NF186 ${ }^{\mathrm{WT}}$-HA or clickable NF186 ${ }^{\mathrm{TAG}}-\mathrm{HA}$ and the AIS length of the untransfected neurons (Fig. 2d).

Considering that neither of the three analyzed NF186 ${ }^{\mathrm{TAG}}-\mathrm{HA}$ amber mutants affected the AIS length, we used an additional parameter to determine the most suitable mutant for click labeling of NF186 in primary neurons. Among other factors, the UAA incorporation efficiency depends on the sequence surrounding the TAG amber codon (Bartoschek et al., 2021). We used a recently developed tool (Bartoschek et al., 2021) to predict the UAA incorporation efficiency rate in the three remaining amber mutants. The iPASS (identification of permissive amber sites for suppression) score was higher for

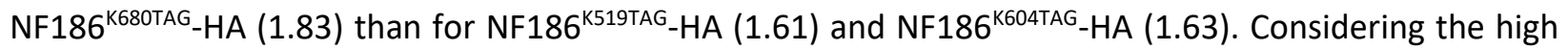
iPASS score and the reproducibly high labeling intensity of NF186 ${ }^{\mathrm{K} 680 \mathrm{TAG}}-\mathrm{HA}$, we decided to use it in future experiments. This allowed us to perform fixed-cell (Fig. 2c) and live-cell confocal imaging of click-labeled NF186 in primary neurons (Fig. 2e). Altogether, these results demonstrate successful application of genetic code expansion and click chemistry for the labeling and imaging of the AIS in living primary neurons.

\section{Click labeling of Nav1.6 channels in living primary neurons}

Once we had established click labeling of NF186 in living primary neurons, we focused on labeling of the alpha subunit of the voltage-gated sodium channel isoform Nav1.6 (encoded by the SCN8A gene). Considering the highly complex structure of Nav1.6 that involves folding a single polypeptide chain of 2000 amino acids (Fig. 3a) into four homologous domains (I to IV), each of which contains six transmembrane segments (S1 to S6), we had to address additional challenges while selecting potential positions for UAA incorporation. First of all, there are many disease-related mutations in the SCN8A gene (Johannesen et al., 2021, Meisler et al., 2021), and there are many conserved regions of Nav1.6 that are crucial for the function of the channel (e.g., the S4 segments of domains I-IV and pore-forming loops; Fig. 3a) (Catterall et al., 2005). These positions/domains had to be avoided when selecting TAG positions 
bioRxiv preprint doi: https://doi.org/10.1101/2022.03.01.480798; this version posted March 3, 2022. The copyright holder for this preprint (which was not certified by peer review) is the author/funder, who has granted bioRxiv a license to display the preprint in perpetuity. It is made available under aCC-BY-NC-ND 4.0 International license.

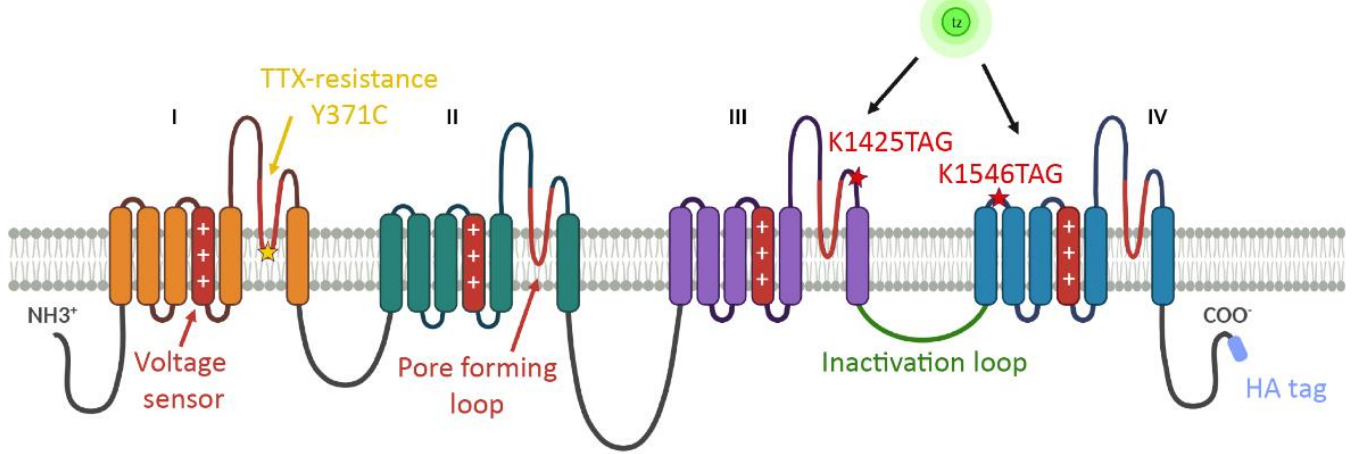

b

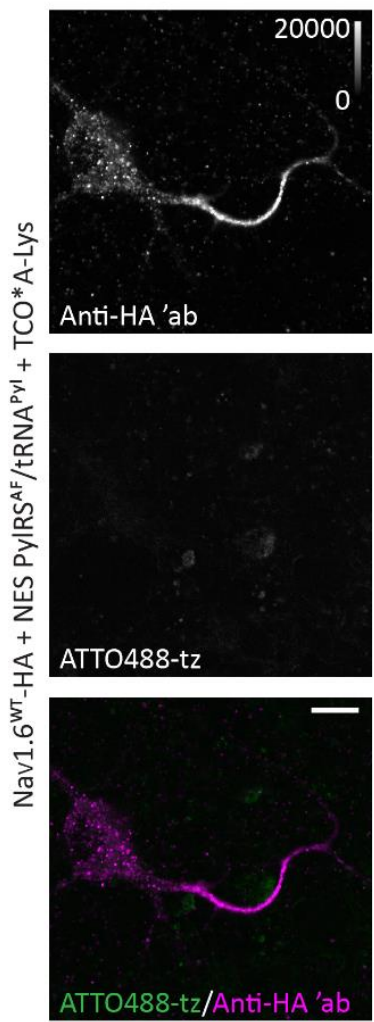

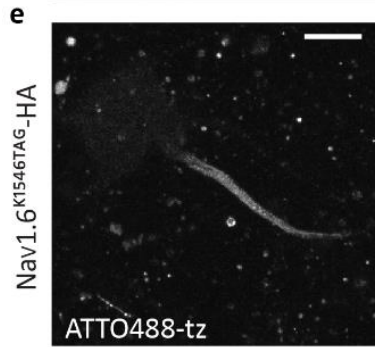

Live-cell imaging
C

K1425TAG
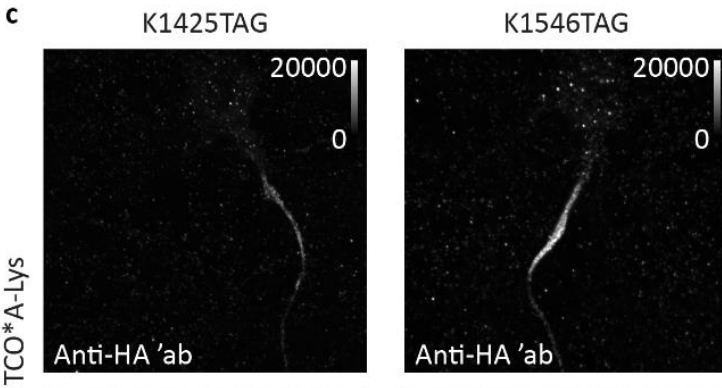

d

K1425TAG
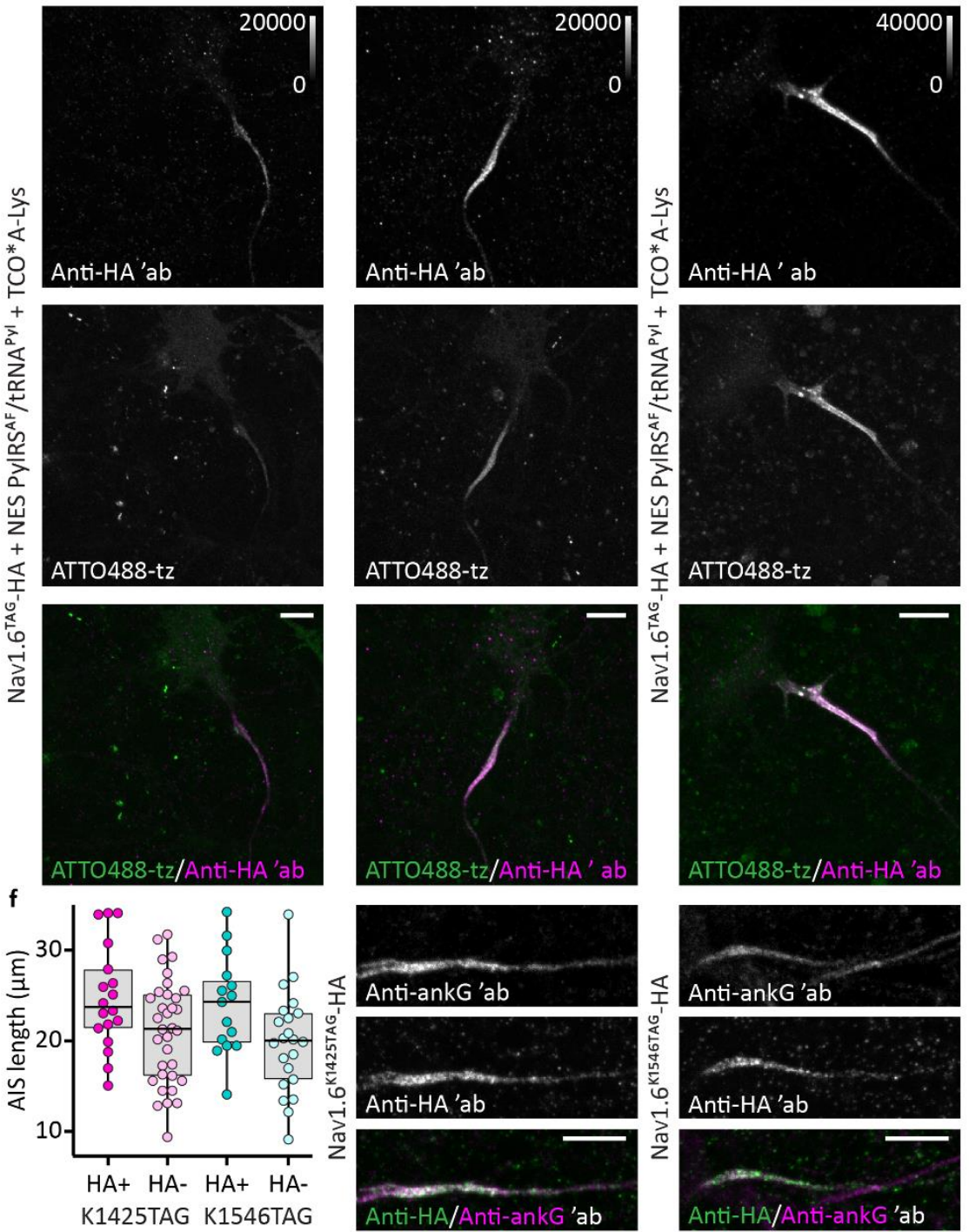

Fig. 3. Genetic code expansion and click labeling of Nav1.6 in living primary neurons. a: Schematic representation of the Nav1.6 alpha subunit with the clickable labeling sites (K1425TAG and K1546TAG; red stars) and the C-terminal hemagglutinin (HA) tag (purple). In addition, the scheme depicts the Y371C mutation (yellow star) that rendered Nav1.6 tetrodotoxin (TTX)-resistant. b- 
Nav1.6 K1425TAG $_{-H A}$, or Nav1.6 K1546TAG $_{-H A}$ in the presence of the UAA TCO*A-Lys. The neurons were transfected with either Lipofectamine 2000 (b,c,e) or Lipofectamine 3000 (d) reagent. Prior to imaging, living neurons were click-labeled with ATTO488-tetrazine (tz), fixed, and immunostained with an anti-HA primary antibody, followed by an Alexa Fluor 555-conjugated secondary antibody. e: Representative confocal image of a living rat neuron at DIV 12 expressing Nav1.6 $6^{\mathrm{K} 1546 T A G}$-HA labeled with ATTO488-tz. f: Distribution of the AIS lengths measured in confocal images of anti-ankG immunostained rat neurons expressing

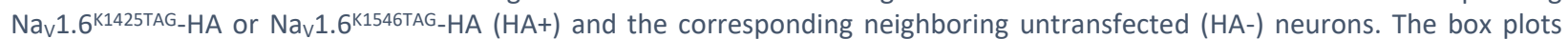
indicate the median (the black lines inside the box), the $25^{\text {th }}$ and $75^{\text {th }}$ percentiles (the box boundaries), and the single data points (the dots). The whiskers lengths are defined by the minimum and maximum data points. One-way ANOVA with Tukey posthoc did not show significant differences between the HA+ and HA- AIS lengths for both clickable mutants $\left(p_{K 1425 T A G}=0.124\right.$, $\mathrm{p}_{\text {K1546TAG }}=0.136$; number $(n)$ of analyzed cells: $n_{\text {K1425TAG HA+ }}=18, n_{\text {K1425TAG HA- }}=34, n_{\text {K1546TAG HA+ }}=15$, and $n_{\text {K1546TAG HA- }}=22$ ). The details of the statistical analysis are shown in Supplementary Table 2. Representative images of rat neurons immunostained with anti-ankG and anti-HA antibodies used for the quantitative analysis are shown in panel f. All images, except for that of Nav1.6 WT-HA (shown in panel b), were taken as Z-stacks and are shown as maximum intensity projections. For the comparison of the Lipofectamine 2000 transfection reagent and Lipofctamine 3000, the brightness and contrast of the panels showing the HA channels (b-d) were linearly adjusted as indicated by the look-up-table (LUT) intensity scale bar. LUT intensity scale bars show the minimum and maximum grey values. Scale bars: $10 \mu \mathrm{m}(\mathbf{b}-\mathbf{f})$. The scheme in panel a was made in BioRender.com.

structure of Nav1.6 is not available, and the alpha subunit of the channel is heavily glycosylated, which made it harder to select TAG positions that would be accessible for the tetrazine dyes. Based on the available literature (Akin et al., 2015, Meisler et al., 2021, O'Brien and Meisler, 2013), we selected two positions (K1425 and K1546) in the extracellular loops of Nav1.6. These positions are located in less conserved regions of the channel, do not participate in the pore formation, opening of the channel, or the regulation of its function; and are not related to any known diseases. We introduced respective TAG mutations into the corresponding sites of a plasmid encoding WT mouse Nav1.6 (Fig. 3a). We also added an HA tag to the $\mathrm{C}$ terminus of Nav1.6, which allowed us to detect full-length proteins and to assess Nav1.6 localization in transfected cells and primary neurons.

Similar to the experiments with NF186, prior to the click labeling of Nav1.6 in primary neurons, we first wanted to establish and optimize click labeling conditions of Nav1.6 ${ }^{\mathrm{TAG}}-\mathrm{HA}$ in neuronal cell lines (Supplementary fig. 5a). We initially probed ND7/23 cells that had been widely used for electrophysiological recordings of $\mathrm{Na}^{+}$currents derived from the recombinant tetrodotoxin (TTX)-resistant variants of Nav1.6 (Meisler et al., 2021, Sharkey et al., 2009). However, our microscopy experiments

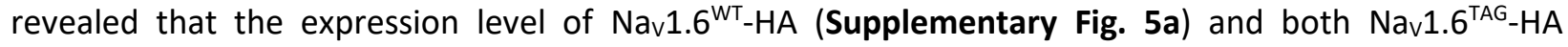
mutants (data not shown) on the membrane of the ND7/23 cells was low. Since the transfection efficiency was low and most of the channels remained inside the cytoplasm, extracellular labeling with the cell-impermeable ATTO488-tz signal was not successful (data not shown). To complement these results functionally, we performed whole-cell patch clamping. We first rendered the WT and clickable Nav1.6-HA constructs TTX-resistant by introducing the previously described Y371C mutation (Fig. 3a) (Leffler et al., 2005, Liu et al., 2019). Most of the measured ND7/23 cells exhibited peak $\mathrm{Na}^{+}$current amplitudes of less than $0.5 \mathrm{nA}$ in the presence of TTX (data not shown), confirming the microscopy results. 
To obtain higher levels of expression, we then tested click labeling conditions in the murine neuroblastoma N1E-115-1 cells (Supplementary Fig. 5b-d). Immunostaining with anti-HA antibody revealed that the expression of Nav1.6 $6^{\mathrm{WT}}-\mathrm{HA}$ on the membrane of the N1E-115-1 cells was higher than on the ND7/23 cells (Supplementary Fig. 5a-c). However, click labeling of both Nav1.6 ${ }^{\mathrm{K} 1425 T A G}-\mathrm{HA}$ and

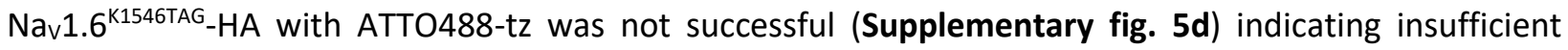
expression of the clickable constructs.

We then reasoned that neuroblastoma cell lines may not be the ideal hosts for click labeling and microscopy studies of $\mathrm{Na} 1.6^{\mathrm{TAG}}$ and that a more native environment is required. Thus, we attempted to express and click label Nav1.6 ${ }^{\text {TAG }}$-HA in primary neurons (Fig. 3b-d and Supplementary Fig. 6). Four days after the transfection, on DIV12, we click labeled living neurons with ATTO488-tz dye and performed immunostaining with an anti-HA antibody. As expected, the WT protein was expressed in the presence and in the absence of the UAA, and click labeling was not detected (Fig. 3b, Supplementary Fig. 6a).

Furthermore, we observed that both Nav1.6 ${ }^{\mathrm{TAG}}-\mathrm{HA}$ mutants were successfully expressed and clicklabeled in the AIS of the neurons (Fig. 3c). In the absence of the UAA, click labeling of amber mutants was not detected (Supplementary Fig. 6b). As shown in the representative images in Fig. 3c, we observed that the expression level and click labeling intensity of Nav1.6 ${ }^{\mathrm{K} 1425 \mathrm{TAG}}-\mathrm{HA}$ were lower than those of

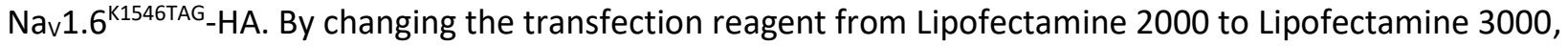
we improved the expression level and consequently, the labeling intensity of Nav1.6 ${ }^{\mathrm{K} 1425 \mathrm{TAG}}-\mathrm{HA}$ (Fig. 3d). However, even though the expression level increased, the number of transfected neurons remained low. In this regard, we noticed that the number of neurons transfected with Lipofectamine 3000 was lower than that of the neurons transfected with Lipofectamine 2000 (5 vs. 15 neurons per well of an eight-well Lab-Tek II chambered cover glass). The high labeling efficiency of Nav1.6 ${ }^{\mathrm{K} 1546 \mathrm{TAG}}$-HA facilitated the confocal imaging of the living click-labeled neurons (Fig. 3e). In conclusion, we successfully applied a combination of genetic code expansion and click chemistry for fluorescent labeling and imaging of the voltage-gated sodium channel isoform Nav1.6 in living primary neurons.

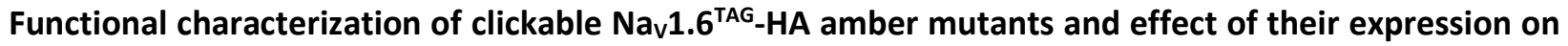
the AIS organization

We next investigated if the AIS structure would be affected by overexpression of the clickable Nav1.6 
2010). As shown in Fig. 3f, the AIS length of neurons expressing Nav1.6 ${ }^{\text {TAG }}$-HA (HA positive) mutants did not significantly differ from that of the untransfected (HA negative) neurons.

Furthermore, we wanted to investigate if incorporation of TCO*A-Lys into Nav1.6 and click labeling had an impact on biophysical properties of the channels or on the nanoscale organization of the AIS. To assess whether functional properties of the UAA-tagged channels are affected, we performed whole-cell patch clamp recordings of $\mathrm{Na}^{+}$currents in N1E-115-1 cells (Fig. 4a-d and Supplementary Fig. 7). Those were

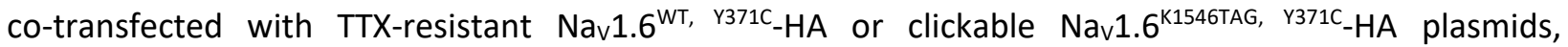

274 NES PyIRS ${ }^{A F} /$ RNA $^{\text {Pyl }}$, and a multigene plasmid that contained mouse $\beta 1$ - and $\beta 2$-subunits and GFP to 275 ensure full functionality of Nav1.6 channels and to identify transfected cells, respectively. The K1546TAG mutation caused a small but significant depolarizing shift $(2.8 \mathrm{mV})$ of the fast inactivation curve, slightly

277 slowed the time course of fast inactivation and accelerated its recovery (Fig. 4a and c; Supplementary Fig.

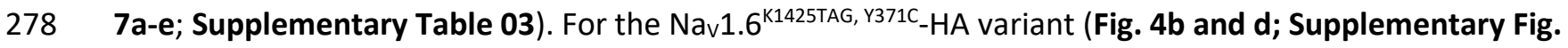

279 7f-j), the number of transfected cells and the expression level were lower than those of Nav1.6 ${ }^{\mathrm{K} 1546 \mathrm{TAG},}$

280 Y371C $-\mathrm{HA}$, corresponding to the reduced $\mathrm{Na}^{+}$currents. To increase the transfection efficiency, we used the commercial PiggyBAC transposase system to stably incorporate the mouse $\beta 1$ - and $\beta 2$-subunits into the genome of the N1E-115-1 cells reducing the number of plasmids needed for transfections of the N1E-115-1 cells. To identify the transfected cells, we generated a plasmid containing the genes encoding Nav1.6 $6^{\mathrm{Y} 371 \mathrm{C}}$ and enhanced GFP (eGFP), separated by a self-cleaving P2A sequence (Nav1.6 ${ }^{\mathrm{Y} 371 \mathrm{C}}-\mathrm{P} 2 \mathrm{~A}-\mathrm{eGFP}$ ). Under these conditions, we acquired a sufficient number of transfected cells and recorded larger $\mathrm{Na}^{+}$ currents. The K1425TAG clickable variant reduced the peak current density compared to the WT channel, whereas changes of other gating parameters were not observed (Fig. 4b and d; Supplementary Fig. 07f-j, Supplementary Table 3). The reduction of the peak current density corresponds well to the reduced expression level of Nav1.6 $6^{\mathrm{K} 1425 \mathrm{TAG}}$ compared to the Nav1.6 ${ }^{\mathrm{WT}}$ protein.

To confirm that the nanoscale periodic organization of the Nav1.6 in the AIS was not affected by the overexpression of our clickable constructs or by the click labeling itself, we next performed direct 292 stochastic optical reconstruction super-resolution microscopy (dSTORM) on the click-labeled and

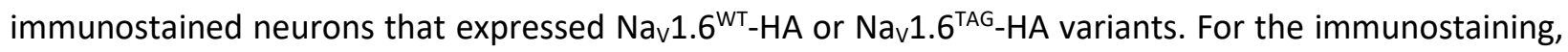


bioRxiv preprint doi: https://doi.org/10.1101/2022.03.01.480798; this version posted March 3, 2022. The copyright holder for this preprint (which was not certified by peer review) is the author/funder, who has granted bioRxiv a license to display the preprint in perpetuity. It is made available under aCC-BY-NC-ND 4.0 International license.

a
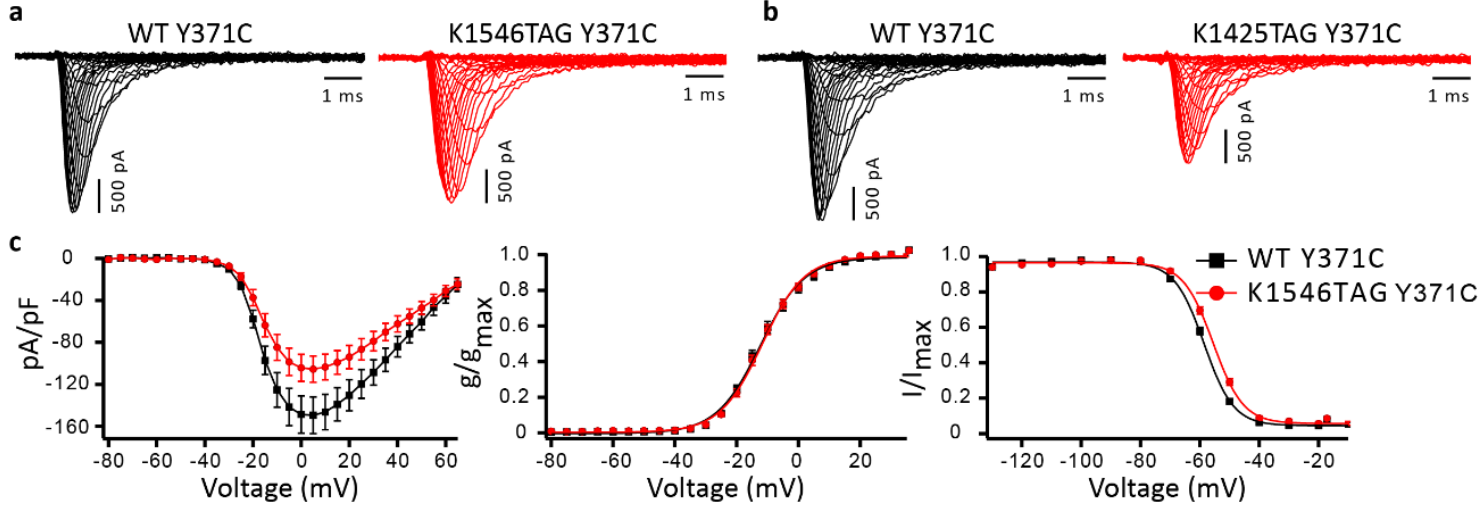

d
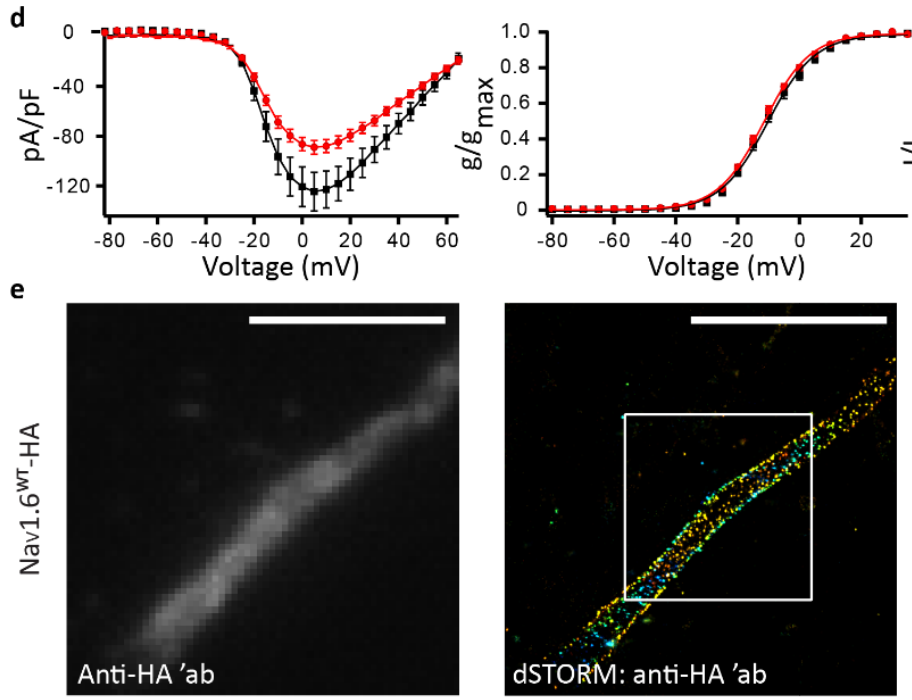

f

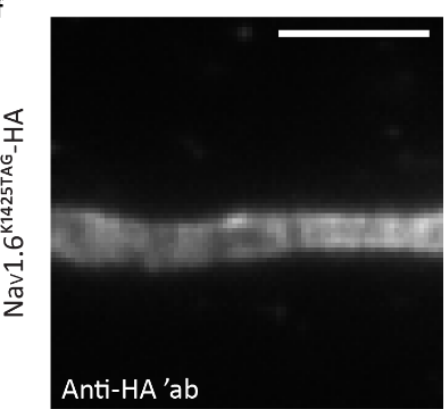

g

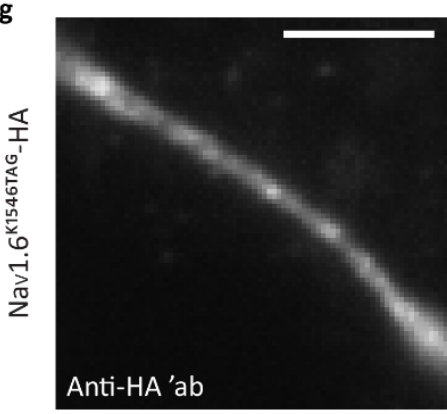

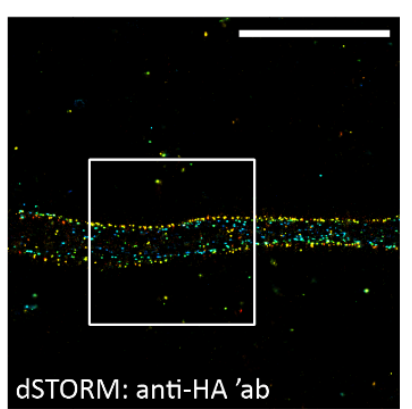

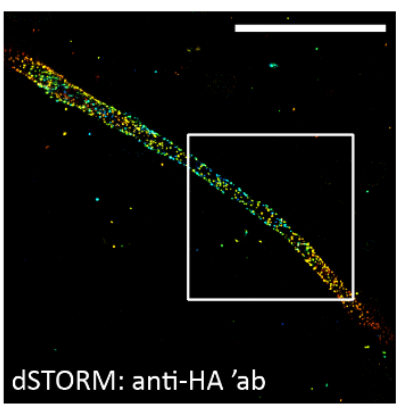

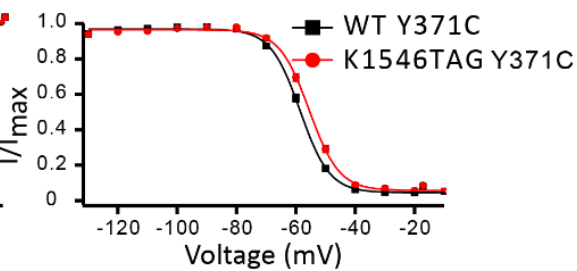
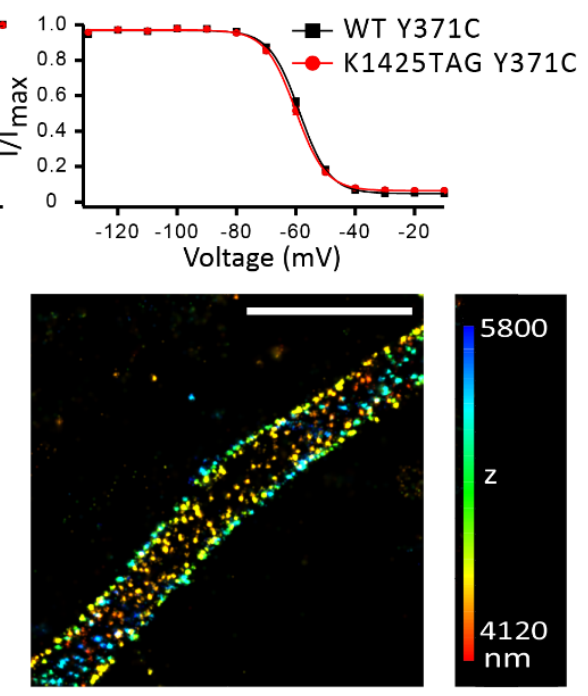

5800

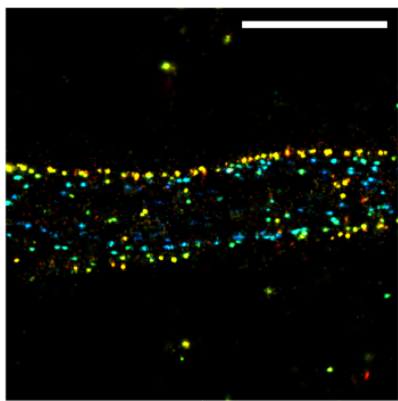

4206

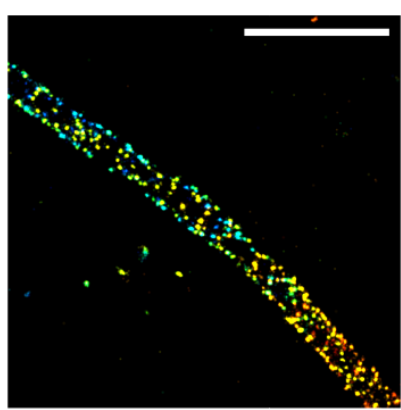

2776

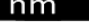


Fig. 4. Functional characterization of clickable Nav1.6 ${ }^{\mathrm{TAG}}$-HA constructs and the effect of their expression on the AIS nanoscale organization. a: Representative $\mathrm{Na}^{+}$current traces obtained from N1E-115-1 cells expressing either Nav1.6 WT, Y371C-HA (black) or

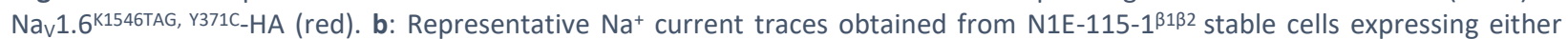

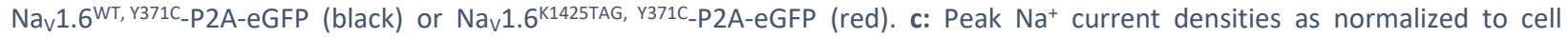
capacitance, were plotted vs. voltage (left panel; number of analyzed $(n)$ cells: $n_{W T}=20$ and $n_{K 1546 T A G}=18$ ), voltage-dependence of activation (middle panel; $n_{W T}=20$ and $n_{K 1546 T A G}=18$ ), and voltage-dependence of fast inactivation (right panel; $n_{W T}=20$ and $n_{\text {K1546TAG }}=17$ ) for K1546TAG (red) vs. WT comparison (black). d: Peak Na+ current densities as normalized to cell capacitance, were plotted vs. voltage (left panel; $n_{W T}=18$ and $n_{K 1425 T A G}=20$ ), voltage-dependence of activation (middle panel; $n_{W T}=18$ and $n_{\text {K1425TAG }}=20$ ), and voltage-dependence of fast inactivation (right panel; $n_{W T}=18$ and $n_{\text {K1425TAG }}=20$ ) for K1425TAG (red) vs. WT comparison (black). The lines represent the Boltzmann functions fit to the data points. Shown are mean \pm standard errors of the mean (SEMs; c, d). Detailed statistical analyses are provided in Supplementary Table 3. e-g: Representative 3D direct stochastic optical (dSTORM) super-resolution images of primary rat cortical neurons at DIV 12-14 expressing NES PyIRSAF/tRNA ${ }^{\text {Pyl }}$ and (e)

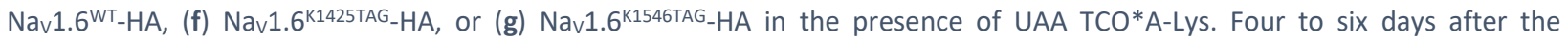
transfection, the neurons were click-labeled, fixed and immunostained with an anti-HA primary antibody and an Alexa Fluor Plus 647-conjugated secondary antibody. The left panels show the TIRF/HILO images of the anti-HA channel, acquired with $647 \mathrm{~nm}$ laser illumination prior to the 3D dSTORM imaging. The middle panels show the corresponding 3D dSTORM images, including a magnified view of the boxed region. The z positions in the 3D dSTORM images are color-coded according to the height maps shown on the right. The height maps contain minimal and maximal z position values. Scale bars: $5 \mu \mathrm{m}$ for the TIRF/HILO and dSTORM images and $2 \mu \mathrm{m}$ for the magnified views of the boxed regions $(\mathbf{e}-\mathbf{g})$.

secondary antibody. Immunostaining of the HA tag fused to WT or clickable Nav1.6 channels allowed us to directly compare them and investigate the effect of TCO*A-Lys incorporation and click labeling on the AIS structure. As there was no obvious difference in the nanoscale organization of the Nav1.6 ${ }^{\mathrm{WT}}-\mathrm{HA}$ or Nav1.6 ${ }^{T A G}-H A$ channels (Fig. 4. e-g), these experiments confirmed that the Nav1.6 overexpression, TCO*ALys incorporation, and click labeling did not affect the nanoscale periodic organization of the sodium channels in the AIS. loss-of-function effect in living primary mouse hippocampal neurons

We next examined whether our click labeling approach in living primary neurons can be used to study the localization of two variants of the human SCN8A gene that cause a generalized epilepsy (T1787P and I1654N). We have recently reported that these two variants strongly reduced the $\mathrm{Na}^{+}$current density in ND7/23 cells and reduced firing in mouse hippocampal neuronal cultures compared to the WT, indicating

327 a loss-of-function (LOF) effect (Johannesen et al., 2021). However, due to the lack of suitable labeling approaches, it has remained unclear whether these variants affect the Nav1.6 channel function or trafficking (thereby leading to a reduced $\mathrm{Na}^{+}$current density). To answer these questions, we introduced the corresponding LOF variants (T1785P and I1652N) in our clickable mouse constructs (mNav1.6 ${ }^{\text {TAG }}$ ).

331 When we transfected N1E-115-1 cells with TTX-resistant versions of the clickable mNav1.6 ${ }^{\text {TAG, Y371C }}$

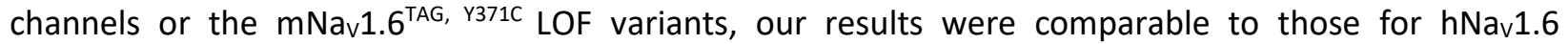


bioRxiv preprint doi: https://doi.org/10.1101/2022.03.01.480798; this version posted March 3, 2022. The copyright holder for this preprint (which was not certified by peer review) is the author/funder, who has granted bioRxiv a license to display the preprint in perpetuity. It is made available under aCC-BY-NC-ND 4.0 International license.

clickable mNav1.6 ${ }^{\mathrm{K} 1425 \mathrm{TAG}}$ or mNav1.6 $6^{\mathrm{K} 1546 \mathrm{TAG}}$ controls (Fig. 5a-b). Hence, we verified that the effect of the

a
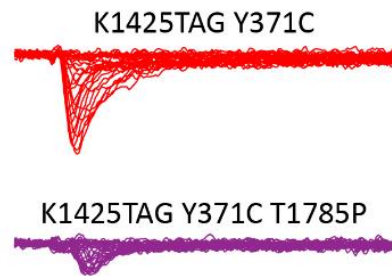

b

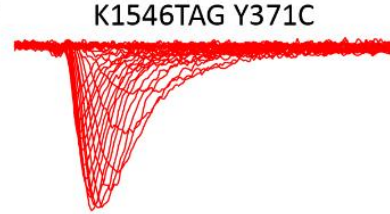

K1546TAG Y371C T1785P
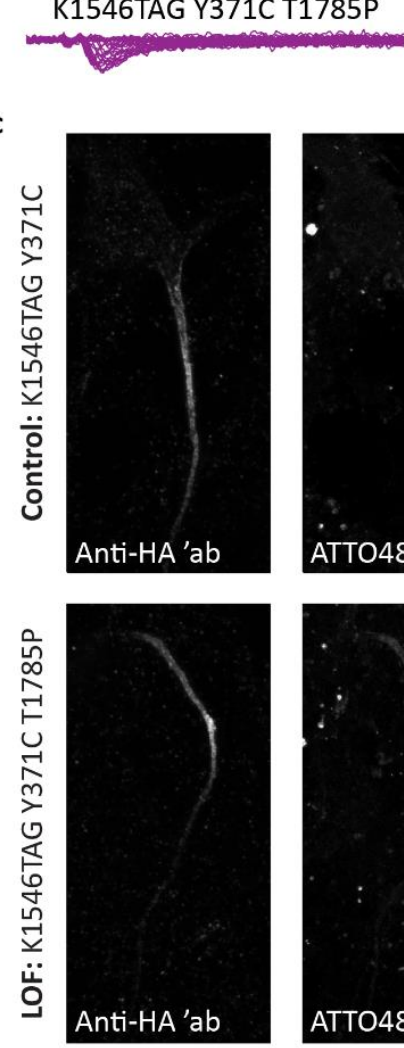

- K1425TAG Y371C

$₫$ K1425TAG Y371C I1652N

$\rightarrow$ K1425TAG Y371C T1785P

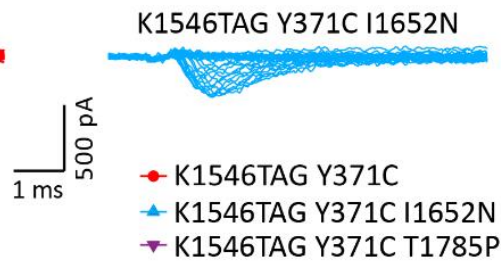

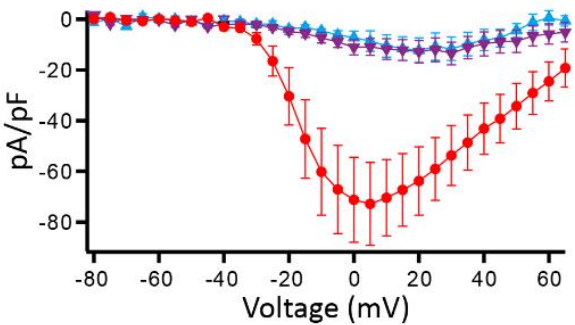

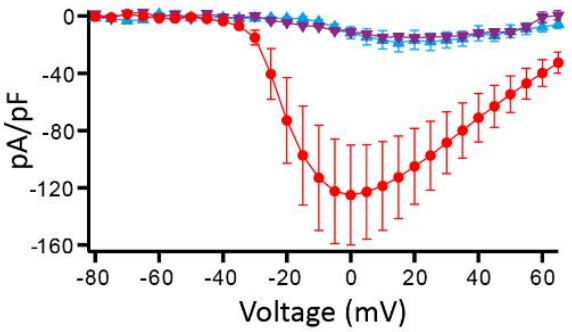

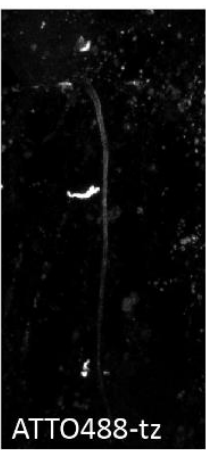

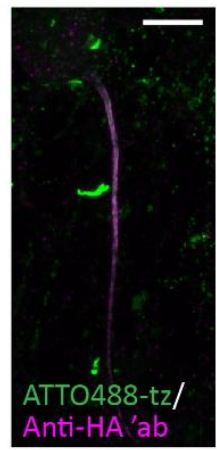

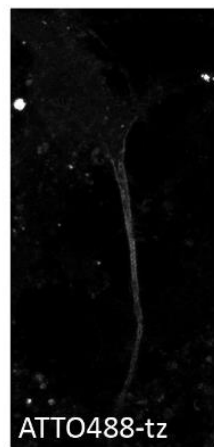
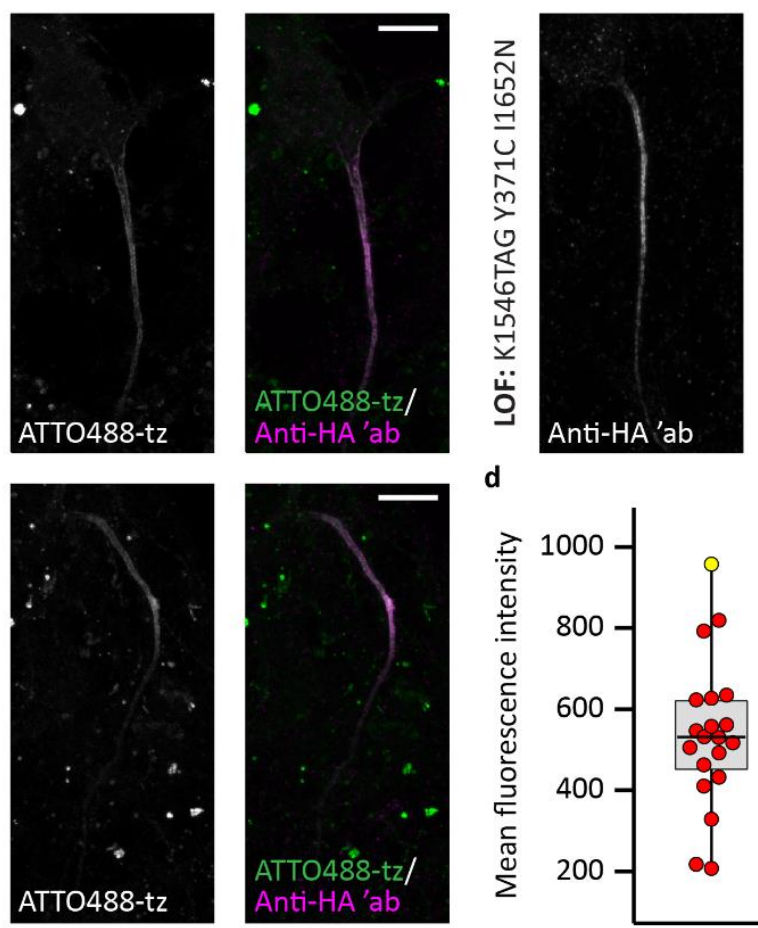

d
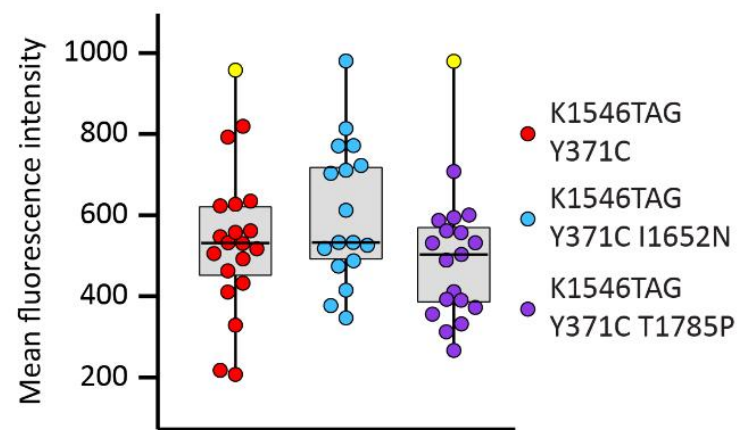

Fig. 5. Click labeling allows localization study of the epilepsy-causing Nav1.6 variants with loss-of-function effect (LOF). a-b: $\mathrm{Na}^{+}$current recordings of mouse Nav1.6 pathogenic variants combined with TAG mutations in N1E-115-1 cells. a: Representative $\mathrm{Na}^{+}$current traces obtained from N1E-115-1 cells expressing Nav1.6 K1425TAG, Y371C-HA (red), Nav1.6 K1425TAG, Y371C, I1652N-HA (blue), or Nav1.6 K1425TAG, Y371C, T1785P-HA (violet). Combined with the K1425TAG Y371C mutant, both the I1652N and T1785P variants significantly reduced the peak $\mathrm{Na}^{+}$current density compared to the K1425TAG Y371C mutant alone (Nav1.6K1425TAG, Y371C-HA: -72.9 $\pm 16.4 \mathrm{pA} / \mathrm{pF}$, number of analyzed cells $(\mathrm{n})=11 ; \mathrm{Nav1.6}$ K1425TAG, Y371C, $11652 \mathrm{~N}_{-} \mathrm{HA}:-12.3 \pm 5.0 \mathrm{pA} / \mathrm{pF}, \mathrm{n}=8, \mathrm{p}=0.0015 ; \mathrm{Nav} 1.6$ K1425TAG, Y371C, T1785P-HA: $-12.7 \pm 4.4 \mathrm{pA} / \mathrm{pF}, \mathrm{n}=12, \mathrm{p}=0.0006$; ANOVA on ranks with Dunn's posthoc test). b: Representative Na+

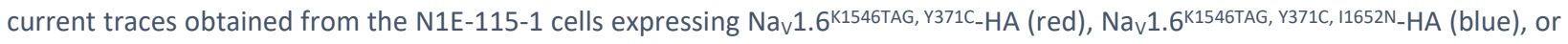
Nav1.6 K1546TAG, Y371C, T1785P-HA (violet). When combined with the K1546TAG Y371C mutant, both the I1652N and T1785P variants significantly reduced the peak Na+ current density compared to the K1546TAG Y371C mutant alone (Nav1.6 ${ }^{\mathrm{K} 1546 T A G}$, Y371C-HA: - 
$\pm 2.0 \mathrm{pA} / \mathrm{pF}, \mathrm{n}=8, \mathrm{p}=0.0014$; ANOVA on ranks with Dunn's posthoc test). Shown are the mean \pm standard errors of the mean (SEMs; a, b). c: Representative confocal images of mouse hippocampal neurons at DIV 12 expressing NES PyIRSAF/tRNA ${ }^{\text {Pyl, }}$

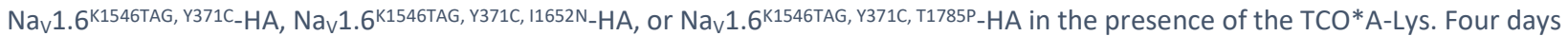
after the transfection, the neurons were click-labeled with ATTO488-tetrazine(tz), fixed, and immunostained with an anti-HA primary antibody and an Alexa Fluor 555-conjugated secondary antibody. d: Distribution of the mean ATTO488-tz fluorescence intensity measured in confocal images of click-labeled mouse neurons expressing the control (Nav1.6 ${ }^{\left.\mathrm{K} 1546 \mathrm{TAG}, \mathrm{Y} 371 \mathrm{C}_{-} \mathrm{HA}\right)}$ or one of

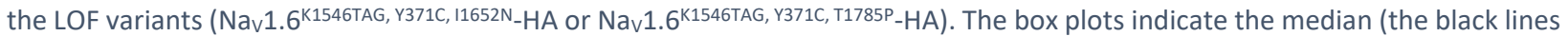
inside the box), the $25^{\text {th }}$ and $75^{\text {th }}$ percentiles (the box boundaries), the single data points (the dots), and the outliers (the yellow dots). The whiskers lengths are defined by the minimum and maximum data points. The non-parametric Kruskal-Wallis test did

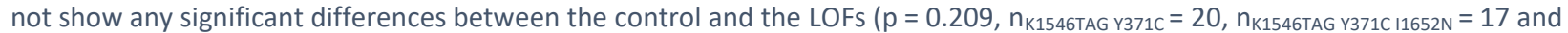
$\mathrm{n}_{\text {K1546TAG Y371C T1785P }}=19$ ). Detailed statistical analyses are provided in Supplementary Table 4. The Z-stack images are shown as maximum intensity projections. Scale bars: $10 \mu \mathrm{m}(\mathbf{c})$.

hNav1.6 LOF variants was the same for the mNav1.6 that we had generated. These constructs were subsequently transfected into primary neurons and click-labeled to visualize the membrane population of the Nav1.6 channels. Since the LOF variants were previously studied in mouse hippocampal neurons (Johannesen et al., 2021), we also used this neuron type for the localization study. We observed that both LOF variants could be labeled extracellularly with click chemistry, suggesting that the channels are expressed in the AIS (Fig. 5c shows representative images for the K1546TAG control and LOF clickable variants). Our quantitative analysis revealed that the AIS fluorescence intensities of both LOF Nav1.6 variants did not significantly differ from that of the control (Fig. 5d, Supplementary Table 4). Therefore, our data suggest that the two variants causing reduced $\mathrm{Na}^{+}$current density affect the channel conductance or opening probability rather than its trafficking to the membrane.

\section{Adeno-associated virus (AAV)-based vectors for delivery of orthogonal translational machinery to primary neurons}

The efficiency of transient transfection in terminally differentiated cells such as primary neurons is generally low. This is especially a problem for transfections of multiple plasmids and large genes, such as those used for click labeling of Nav1.6. To overcome this limitation, we developed AAV vectors as tools for delivering NESPyIRS ${ }^{A F}$ and tRNA ${ }^{\text {Pyl }}$ to primary neurons. AAVs had been used in a proof-of-concept study that showed amber codon suppression of a fluorescent reporter protein in mouse neurons (Ernst et al., 2016).

To find a suitable AAV capsid for neuron transduction, we first probed the engineered variants AAV9A2 and AAV7A2. In previous work, we had generated these variants by inserting the small peptide NYSRGVD (called A2) into exposed regions on the capsids of the AAV serotypes AAV7 or AAV9, respectively, and we found that they efficiently transduced a wide variety of cell types (Borner et al., 2020). In this study, we transduced primary neurons with AAV9A2 or AAV7A2 that bore a fluorescent reporter which consisted of 
bioRxiv preprint doi: https://doi.org/10.1101/2022.03.01.480798; this version posted March 3, 2022. The copyright holder for this preprint (which was not certified by peer review) is the author/funder, who has granted bioRxiv a license to display the preprint in perpetuity. It is made available under aCC-BY-NC-ND 4.0 International license.

AAV\#1:

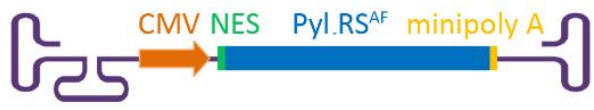

AAV\#2:

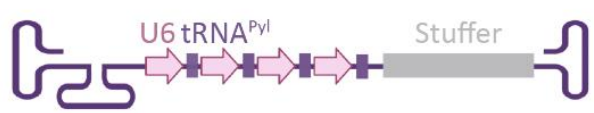

Plasmid\#1:

CMV

$m S C N 8 A^{\text {K1425TAG }}$

HA

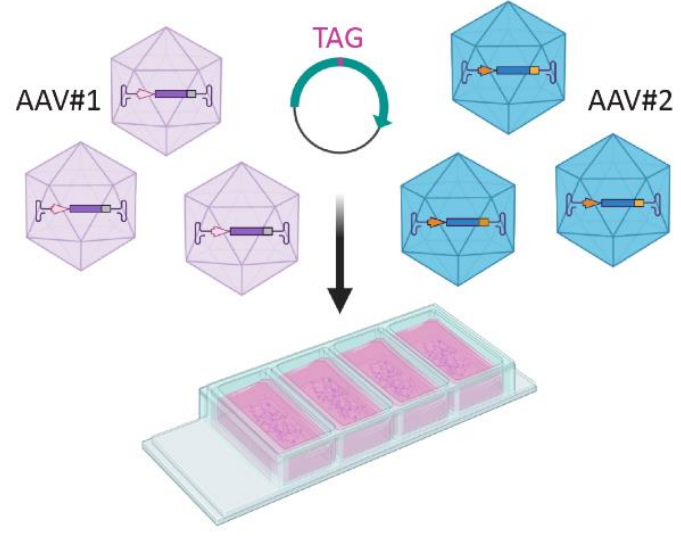

b
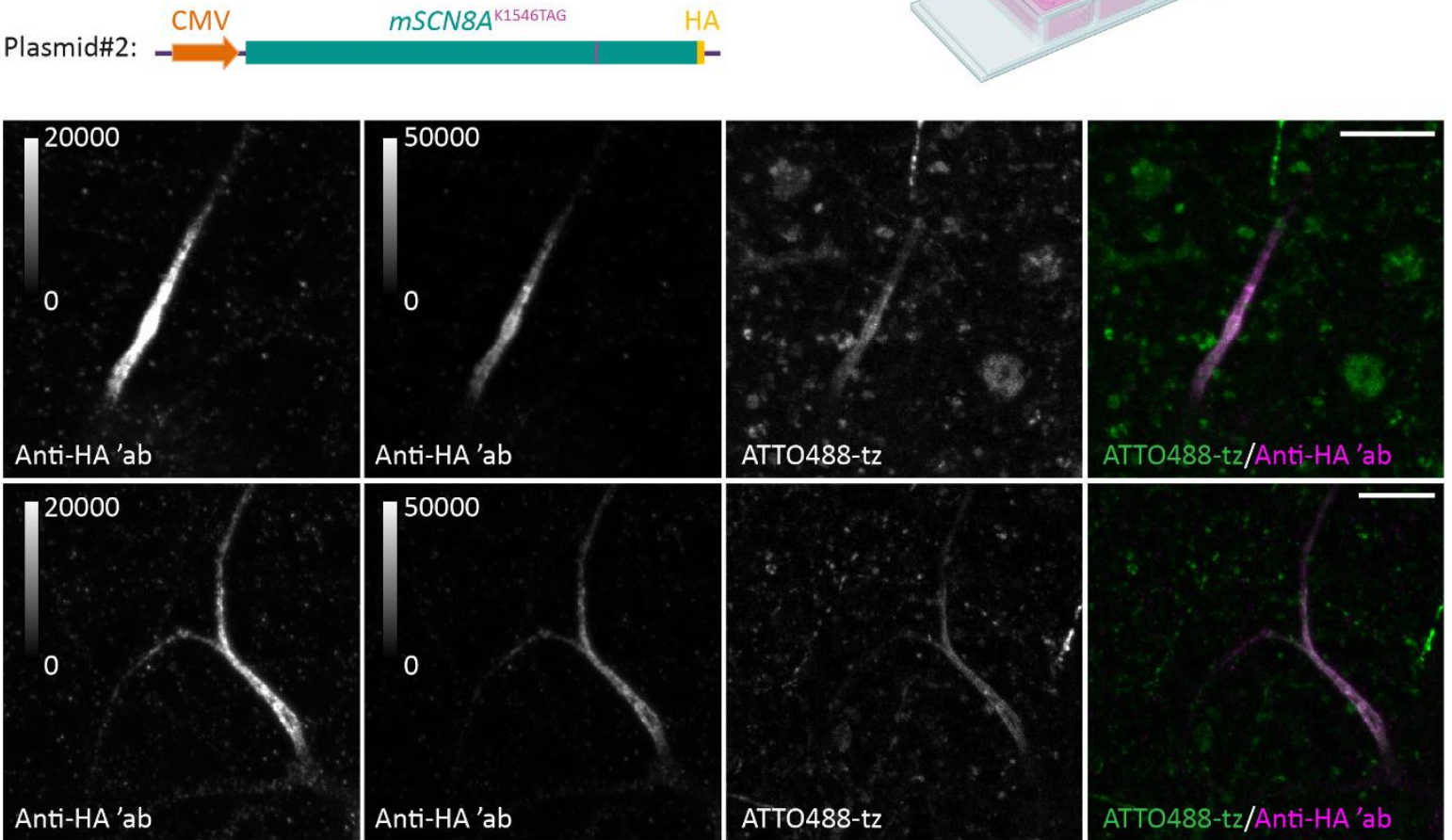

c
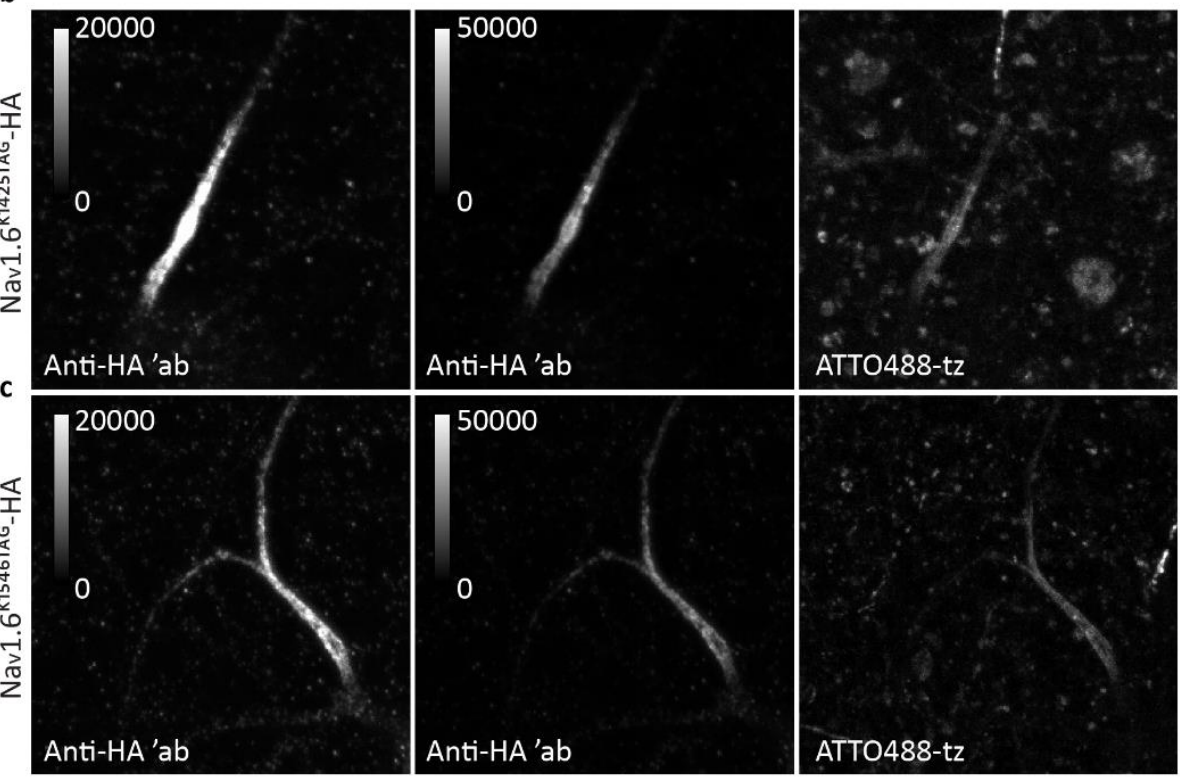

ATTO488-tz

ATTO488-tz/Anti-HA 'ab

Fig. 6. Adeno-associated virus (AAV)-based vectors are an efficient tool for delivery of orthogonal translational machinery for click labeling of Nav1.6 in primary neurons. a: Schematic representation of a combination of plasmid transfection and transduction with AAV9A2 viral vectors in primary rat cortical neurons. b-c: Representative confocal images of neurons at DIV 11 expressing NES PyIRSAF/4xtRNA ${ }^{\text {Pyl }}$ and (b) Nav1.6 ${ }^{\text {K1425TAG }}$-HA or (c) Nav1.6 ${ }^{\text {K1546TAG }}$-HA in the presence of UAA TCOA*-Lys. The neurons were transfected with clickable Nav1.6 and transduced with orthogonal translational machinery components: NES PyIRSAF (AAV\#1) and four copies of tRNA ${ }^{\text {Pyl }}$ (AAV\#2) at DIV 8. Prior to imaging, living neurons were click-labeled with ATTO488tetrazine (tz), fixed, and immunostained with an anti-HA primary antibody, followed by an Alexa Fluor 555 secondary antibody. The Z-stack images are shown as maximum intensity projections. For comparison with the transfected neurons (as shown in Fig. $3 \mathrm{c})$, the brightness and contrast of the panels showing the HA channel were linearly adjusted to show the same display range (020,000). In addition, they were adjusted to show a broader display range (0-50,000), as indicated by the look-up-table (LUT) intensity scale bar. LUT intensity scale bars show the minimum and maximum grey values. Scale bars: $10 \mu \mathrm{m}(\mathbf{b}-\mathbf{c})$.

been transduced with the AAV9A2 variant started producing mCherry earlier and at slightly higher levels than the neurons that had been transduced with the AAV7A2. We then co-transduced neurons with

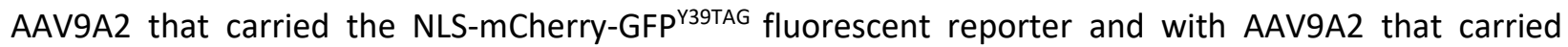
different combinations of promoters and genes for UAA incorporation (Supplementary Fig. 8a). Tested 
promoters and genes included multiple copies of $\mathrm{tRNA}^{\mathrm{Pyl}}$ and the mutant $\mathrm{eRF} 1^{\mathrm{E5} 5 \mathrm{D}}$ elongation factor (Schmied et al., 2014) which had been used to increase the efficiency of amber codon suppression, as well as conventional and minimal CMV promoters for the expression of NESPyIRS ${ }^{\mathrm{AF}}$ and eRF1 ${ }^{\mathrm{ES} 5 \mathrm{D}}$. These

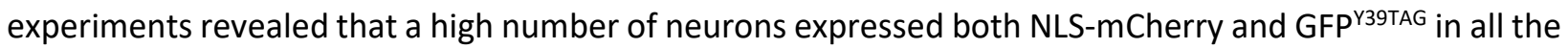
tested conditions (Supplementary Fig. 8b), which confirmed that our AAVs can be used for successful amber codon suppression of a reporter fluorescent protein. Subsequently, we tried to use these AAV vectors for click labeling of Nav1.6. Since the AAVs have a limited packaging capacity, we were not able to pack large $m S C N 8 A$ genes into an AAV. Instead, for click labeling of Nav1.6, we combined transfection of the clickable Nav1.6 ${ }^{\text {TAG }}$ plasmids and transduction with the AAV9A2 vectors that carried components for UAA incorporation. Similar to the experiments with the mCherry-GFP ${ }^{Y 39 T A G}$ reporter (Supplementary Fig. 8), we transduced neurons with combinations of AAV9A2 vectors that carried different orthogonal translational machinery genes. Although all the tested combinations resulted in successful click labeling (data not shown), a combination of AAVs that bore NES PyIRS ${ }^{A F}$ and four copies of tRNA ${ }^{\text {Pyl }}$ showed the lowest background (Fig. 6). In addition, the microscopy experiments revealed that the expression level

(Fig. 6b) and the number of neurons that expressed clickable Nav1.6 were higher than in the case of the transfections ( 50 transduced neurons vs. $\sim 5-15$ transfected per well of an eight-well Lab-Tek II chambered cover glass). In conclusion, we developed AAV viral vectors that enabled successful genetic code expansion and click labeling in primary neurons.

\section{7}

\section{Discussion}

Since the AIS organization is highly complex and unique, care should be taken when choosing a livecell labeling method for microscopy studies of its components. In this study, we combined genetic code expansion and bioorthogonal click chemistry to perform direct fluorescent labeling of two AIS components: the cell adhesion molecule NF186 and the voltage-gated ion channel Nav1.6. The main advantage of our labeling approach is that the small fluorescent dye is directly attached to the proteins of interest in living neurons. Importantly, a clickable UAA in a complex with a dye is much smaller ( 0.5$2 \mathrm{kDa}$ ) than fluorescent proteins $(\sim 30 \mathrm{kDa})$ and antibodies (over $150 \mathrm{kDa})$. Therefore, the protein of interest is modified in a minimally invasive way. This is of particular importance for the labeling of Nav, where a single mutation can severely impair the function of the channel (Johannesen et al., 2021, Meisler et al., 2021, Sole and Tamkun, 2020). Since the UAAs are introduced site-specifically into the protein of interest, positions and domains that are important for the function of the target protein can be avoided. For these reasons, click chemistry represents a powerful approach for live-cell labeling of AIS components. 
Although click labeling has been used for other smaller neuronal proteins and cytoskeleton (Arsić et al., 2022, Bessa-Neto et al., 2021), NF186 and Nav1.6 are challenging targets due to their large size and spatially restricted expression at the AIS. For technical reasons, we first established click labeling of the smaller AIS component, NF186 (186 kDa). Although we showed that several clickable variants were suitable for fluorescent labeling, we continued using the K680TAG variant due to its bright labeling. Labeling of Nav1.6 was more challenging due to its complex structure and larger size (260 kDa). We unsuccessfully attempted click labeling in two host cell lines before we were able to establish it in primary neurons. For this purpose, we probed two positions in extracellular loops of Nav1.6: K1546 and K1425. It was reported previously that incorporation of the 17-amino-acid-long BAD domain at position K1546 minimally affected the function of Nav1.6 (Akin et al., 2015). Akin et al. showed that the current density measured in rat hippocampal neurons was reduced, while other biophysical parameters measured in ND7/23 cells were not affected. In this study, we observed that both clickable variants reduced the current density, although this reduction appeared statistically significant only in the case of Nav1.6 $6^{\mathrm{K} 1425 \mathrm{TAG}}$. The reduction in the current density of clickable Nav1.6 is not surprising due to the fact that suppression of amber codons is not 100\% efficient (Arsić et al., 2022, Bartoschek et al., 2021, Nikic-Spiegel, 2020). In addition, we observed a small shift of $2.8 \mathrm{mV}$ in the inactivation curve of the clickable Nav1.6 ${ }^{\mathrm{K} 1546 \mathrm{TAG}}$ variant, while the Nav1.6 ${ }^{\mathrm{K} 1425 \mathrm{AG}}$ clickable variant did not alter the biophysical properties of the channel. However, the expression and click labeling efficiency of K1425TAG were lower than those of K1546TAG. Hence, we tried to increase the expression level of K1425TAG, first by changing the transfection reagent and later by using AAV-based viral vectors to deliver orthogonal translational machinery. However, even with higher expression, click labeling of K1425TAG was weaker than click labeling of K1546TAG, most likely due to the reduced accessibility of the UAA for tetrazine-dye. That is why we continued to use K1546TAG mutant. Even though AAV vectors increased the expression of our clickable Nav1.6 constructs, it is important to address their limitations. Due to the AAV's limited packaging capacity of $\sim 5 \mathrm{~kb}$, we used multiple AAVs to deliver different components of the orthogonal translational machinery. We are currently attempting to overcome this limitation by testing AAV variants with minimal promoters that allow packaging of all necessary components in one AAV. Furthermore, we were not able to pack the large 457 mSCN8A gene into an AAV. To avoid the combination of plasmid transfection and AAV transduction, 458 baculoviruses that have a high packaging capacity could be used instead. They were used previously for 459 genetic code expansion of a fluorescent reporter protein in cells and ex vivo mouse brain slice culture 460 (Chatterjee et al., 2013, Zheng et al., 2017). 
In summary, we developed a minimally invasive approach for labeling of NF186, as well as for WT and epilepsy-causing pathogenic Nav1.6 channels, with small fluorescent dyes in living neurons. UAA-based minimal tags offer the opportunity to study localization and trafficking of NF186 and Nav1.6 in developing, mature, healthy, or injured neurons. Thanks to its compatibility with live-cell imaging, this labeling approach will provide new insights into the dynamics and plasticity of these proteins. Furthermore, the combination of two different tetrazine dyes (Arsić et al., 2022) or two different click reactions (Nikic et al., 2014) in a pulse-chase manner can be used to study different populations of NF186 or sodium channels. Moreover, in addition to conventional imaging, the small size of the labeling tag and the variety of available tetrazine dyes make click labeling particularly suitable for super-resolution imaging such as dSTORM or stimulated emission depletion (STED) microscopy (Arsić et al., 2022, Bessa-Neto et al., 2021).

471 We also showed that our labeling approach can be used not only for different AIS components but also

472 across different neuronal types (i.e., mouse hippocampal neurons and rat cortical neurons), thereby

473 strengthening the potential of this method. Furthermore, click chemistry labeling can be easily adjusted

474 for other neurofascin and voltage-gated sodium channel isoforms, including different disease-associated variants. This method can also be established for click labeling of other AIS and nodes of Ranvier proteins, 476 including ion channels, for which antibodies, fluorescent protein fusions, and other labeling tags cannot

477 be used. Finally, we developed AAV-based viral vectors to more efficiently deliver the components

478 required for genetic code expansion to primary neurons. Together with the availability of natural or 479 synthetic AAV capsid variants as well as hybrid parvoviral vectors with different cell type-specificities 480 (Fakhiri and Grimm, 2021, Grimm and Zolotukhin, 2015), this will facilitate application of click chemistry481 based protein engineering in more complex biological systems, such as organotypic slice cultures, 482 organoids and animal models.

\section{Acknowledgments}

484 We would like to thank Katja Widmaier for her excellent technical assistance and all the members of the 485 Nikić-Spiegel group for their support, Dr. Rainer Spiegel for his advice on statistical analysis, the 486 laboratories of Dr. Vann Bennett, Dr. Michael Davidson, and Dr. Rosalyn Adam for sharing plasmids that 487 were obtained through Addgene, and the laboratory of Dr. Matthew Grubb and Dr. Christophe Leterrier 488 for sharing MATLAB scripts and Fiji/ImageJ macros sets for AIS quantification. We would also like to thank 489 Dr. Edvard Lemke for the gift of NES PyIRS ${ }^{A F} /$ RRNA $^{\text {Pyl, }}$, eRF1 ${ }^{\text {E55D }}$, and (NLS)-mCherry-GFP ${ }^{\mathrm{Y} 39 T A G}$ plasmids and 490 Dr. Jason Chin for the gift of the plasmid that contains $4 x t R N A^{\text {Pyl }}$ cassette. We are also grateful to George 491 Philippos for his help with the mutagenesis of NF186-HA. This study was supported by the Emmy Noether 
Programme (project number 317530061 to I.N.-S.) of the German 1061 Research Foundation (DFG) and the Werner Reichardt Centre for Integrative Neuroscience (Ministry of Science Baden-Württemberg and former Excellence Cluster EXC307 from the DFG). This work was moreover enabled by funding from the DFG Collaborative Research Center SFB1129 (project number 240245660 to D.G.) and the Cluster of Excellence CellNetworks (EXC81 to D.G.). The electrophysiological experiments were supported by the Research Unit FOR-2715 of the DFG (grant Le1030/15-2 to H.L.).

\section{Author contributions}

N.S. designed and performed the experiments that involved molecular biology, transfection of neuronal cells and primary neurons, click labeling, and microscopy. N.S. and I.N.- S. analyzed the click labeling and microscopy data. Y.L. and H.L. designed, performed, and analyzed the experiments that involved electrophysiological recordings of Nav1.6. A.A. optimized the conditions for the transfections and the amber codon suppression in the primary neurons and contributed to the data analysis. N.M. and D.G. designed and provided the AAV viral vectors. H.Lyu. and N.Z. performed the electrophysiological recordings of the loss-of-function Nav1.6 variants in the N1E-115-1 cells. N.S. and I.N.- S. prepared the figures (with the help of Y.L.) and wrote the manuscript with the inputs of all the authors. I.N.-S. conceived and supervised the project. All the authors reviewed and approved the final manuscript.

\section{Competing interests statement}

D.G. is a co-founder of AaviGen GmbH. All the other authors declare no competing interests.

\section{Materials and Methods}

\section{Plasmids, cloning, and mutagenesis}

For click labeling of NF186, we used a plasmid that contained a rat Nfasc gene with a hemagglutinin (HA) tag at the $\mathrm{C}$ terminus. This construct was generated from a plasmid that contained a wild-type (WT) rat Nfasc gene expressed from the cytomegalovirus (CMV) promoter (a gift from Vann Bennett, Addgene plasmid \# 31061; http://n2t.net/addgene:31061; RRID: Addgene_31061) (Zhang et al., 1998) by moving the $\mathrm{HA}$ tag from the $\mathrm{N}$ terminus to the $\mathrm{C}$ terminus. To delete the $\mathrm{HA}$ tag from the $\mathrm{N}$ terminus, we used the Quick Change II XL site-directed mutagenesis kit (Agilent Technologies, cat. no. 200522) following the manufacturer's instructions. In the resulting construct, the HA tag was added to the C terminus via polymerase chain reaction (PCR)-mediated cloning using the Apal and Notl restriction sites (resulting plasmid: CMV-NF186 ${ }^{\text {WT}}-\mathrm{HA}$ ). Clickable NF186-HA mutants (CMV-NF186 ${ }^{\text {TAG }}$-HA) were generated by 
522 introducing amber stop (TAG) codons in the Nfasc gene of the original Addgene plasmid at positions K534

523 and $\mathrm{K} 680$, or by modifying CMV-NF186 ${ }^{\mathrm{WT}}$-HA by introducing TAG codons in the Nfasc gene at positions

$524 \mathrm{~K} 519, \mathrm{~K} 571, \mathrm{~K} 604$, or K809. All modifications were introduced by PCR-based site-directed mutagenesis. In

525 the final experiments, CMV-NF186 ${ }^{\text {WT/TAG }}$-HA was used. For click labeling of NF186 in primary neurons, the

526 CMV promotor was excised from CMV-NF186 ${ }^{\text {WT }}$-HA and six CMV-NF186 ${ }^{\text {TAG }}$-HA plasmids and replaced with

527 the human neuron-specific enolase 2 promotor (hNSE) by using the Asel and Bglll restriction sites. The

528 hNSE promoter was amplified from the pGL3 NSE plasmid (a gift from Rosalyn Adam, Addgene plasmid \# 11606; http://n2t.net/addgene:11606; RRID: Addgene_11606) (Kim et al., 2004). We used One Shot ${ }^{\mathrm{TM}}$ TOP10 Electrocompetent E. coli (Thermo Fisher Scientific, cat. no. C40452) in all the experiments that

531 involved mutagenesis, cloning, and amplification of the NF186 plasmids, except for the experiment that

532 involved the deletion of the HA tag, where we used the XL 10-Gold Ultracompetent Cells (Agilent, cat. no. 200315) provided with the mutagenesis kit.

Mouse 654 base pair-long SCN1B (mSCN1B, Clone ID: OMu07915D ORF clone, accession no. NM:011322.2) and mouse 558 base pair-long SCN2B (mSCN2B, clone ID: Omu42415D ORF clone, accession no. XM:006510629.3 ORF sequence) cDNAs cloned into the pcDNA3.1+/C-(k)-DYK vectors were obtained from GenScript. For the electrophysiological recordings of the $\mathrm{Na}^{+}$currents, we made a multigene plasmid that contained monomeric enhanced-GFP (mEGFP) and the $m S C N 1 B$ and $m S C N 2 B$ genes using the MultiBacMam system ${ }^{\mathrm{TM}}$ kit (Geneva Biotech). We first cloned $m S C N 1 B$ into the pACEMam2 acceptor vector (pACE Mam2 $\beta 1$ ) and $m S C N 2 B$ into the pMDS donor vector (pMDS $\beta 2$ ) using 541 the Nhel and Kpnl restriction sites, and then we cloned mEGFP into the pMDC donor vector 542 (pMDC mEGFP) using the BamHI and Xbal restriction sites. The donor and acceptor vectors were 543 components of the MultiBacMam system ${ }^{\mathrm{TM}}$ kit, while cDNA encoding for mEGFP was amplified from the 544 mEGFP-N1 plasmid (a gift from Michael Davidson, Addgene plasmid \#54767; 545 http://n2t.net/addgene:54767; RRID: Addgene_54767). The multigene construct

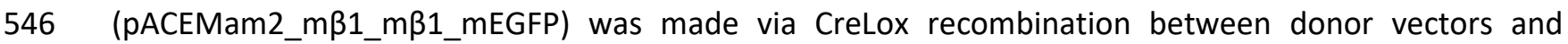
547 acceptor vector, following the manufacturer's instructions. The donor vectors were propagated in pirHC 548 cells (Geneva Biotech), while all the other plasmids were propagated in One Shot ${ }^{\mathrm{TM}}$ TOP10 549 Electrocompetent ${ }^{\mathrm{TM}}$ E. coli. For the generation of the N1E- $115^{\beta 1 \beta 2}$ stable cell lines, we cloned mSCN1B or 550 mSCN2B into the PiggyBAC-CMV-MCS-EF1-Puro cDNA/miRNA Cloning and Expression vector 551 (BioCat, cat. no. PB510B-1-SBI) using the Nhel and Notl restriction sites (PiggyBAC m $\beta 1$, PiggyBAC $\mathrm{m} \beta 2$ ). 
Mouse voltage-gated sodium channel 5934 base pair-long genes ( $m S C N 8 A^{\mathrm{WT}}, m S C N 8 A^{\mathrm{K} 1425 T A G}$, and $m S C N 8 A^{\text {K1546TAG }}$ were synthetized by GenScript (mSCN8A; transcript variant 1, NCBI Reference Sequence: NM_001077499.2) and cloned into pcDNA3.1-P2A-eGFP vectors using EcoRI and Xbal restriction sites. The final plasmids contained the $m S C N 8 A$ gene, followed by the Xbal restriction site, self-cleaving (22 amino acids) P2A sequence, eGFP, and TAA stop codon (Nav1.6-P2A-eGFP). For the establishment of Nav1.6 click labeling, P2A-eGFP was excised and replaced with the HA tag using the Apal and Xball restriction sites (Supplementary Table 5). In the final plasmids, the open-reading frame (ORF) contained the mSCN8A gene, followed by the HA tag and a TAA stop codon ( $\mathrm{Nav1.6-HA).} \mathrm{The} \mathrm{HA} \mathrm{tag} \mathrm{oligonucleotide}$ strand was synthetized by Sigma-Aldrich as two complementary single-stranded oligonucleotides (Supplementary Table 5). For the electrophysiological recordings of the $\mathrm{Na}^{+}$currents, the Nav1.6-P2A-eGFP and Nav1.6-HA plasmids were rendered TTX-resistant by introducing the previously described Y371C point mutation into the $m S C N 8 A^{\mathrm{WT}}$ and $m S C N 8 A^{\mathrm{TAG}}$ genes (Leffler et al., 2005, Liu et al., 2019). To study the localization of the LOF mNav1.6-HA variants, we introduced I1652N or T1785P mutations (Johannesen et al., 2021) in the $m S C N 8 A^{\mathrm{WT}, \text { Y371C }}, m S C N 8 A^{\mathrm{K} 1425 T A G, \text { Y371C }}$, and $m S C N 8 A^{\mathrm{K} 1546 \mathrm{TAG}, \mathrm{Y} 371 \mathrm{C}}$ genes. Mutagenesis of $m S C N 8 A$ was performed using the Quick Change II XL site-directed mutagenesis kit. In the experiments that involved cloning, mutagenesis, and amplification of $m S C N 8 A$, we used chemically-competent XL-10 Gold Ultracompetent cells. All the steps that involved propagation of Nav1.6 in bacteria were performed at $27-28^{\circ} \mathrm{C}$ (Feldman and Lossin, 2014, O'Brien and Meisler, 2013) to avoid the introduction of additional mutations and rearrangements of $m S C N 8 A$.

For the incorporation of TCO*A-Lys into of NF186 ${ }^{\text {TAG }}-\mathrm{HA}$ and Nav1.6 $6^{\mathrm{TAG}}-\mathrm{HA}$, we used a recently described pcDNA3.1/Zeo(+) plasmid that contained the codon-optimized Methanosarcina mazei-derived Y306A/Y384F (AF) double-mutant pyrrolysyl (Pyl) tRNA synthetase fused to a nuclear export signal (NES PyIRS ${ }^{\text {AF }}$ ) and its cognate amber codon suppressor tRNA ${ }^{\text {Pyl }}$ (Arsić et al., 2022). For electrophysiological recordings of $\mathrm{Na}^{+}$currents, we transfected N1E-115-1 cells with the codon-optimized NES PyIRSAF/tRNA ${ }^{\mathrm{Pyl}}$ and WT or K1546TAG Nav1.6 plasmids, while we transfected the N1E-115-1 ${ }^{\beta 1 \beta 2}$ cells with NES PyIRS ${ }^{A F} /$ tRNA $^{\text {Pyl }}$, which was a gift from Dr. Edward Lemke (EMBL, Heidelberg, and IMB, Mainz), and with WT or the K1425TAG Nav1.6 plasmids.

All the modifications introduced into the abovementioned plasmids were confirmed by sequencing. For modifications of Nav1.6, the entire mSCN8A ORFs were sequenced to confirm that there were no additional mutations or rearrangements before they were used for the transfections. The mutagenesis primers, cloning primers, and oligonucleotide sequences are provided in Supplementary Table 5. 


\section{UAAs, tetrazine-dye derivatives, and antibodies}

584

585

586

587

588

589

590

591

592

593

594

595

596

597

598

599

600

601

602

603

604

605

606

607

608

609

610

611

612

We used the trans-cyclooct-2-en-L-lysine (TCO*A-Lys; Sirius Fine Chemicals, SICHEM, cat. no. SC-8008) UAA. A 100 mM stock solution (in 0.2 M NaOH and 15\% DMSO) of TCO*A-Lys was diluted 1:4 in 1 M HEPES (Thermo Fisher Scientific, cat. no. 15630056), and added to the medium at a final concentration of $250 \mu \mathrm{M}$. For click labeling of NF186-HA and Nav1.6-HA, we used ATTO488-tetrazine (ATTO488-tz; Jena Bioscience, cat. no. CLK-010-02). A 500 MM ATTO488-tz stock solution in DMSO was diluted in a warm culture medium at a final concentration of $1.5 \mu \mathrm{M}, 3 \mu \mathrm{M}$ or $5 \mu \mathrm{M}$.

The primary antibodies were as follows: mouse anti-HA tag (2-2.2.14) antibody (1:1000; Thermo Fisher Scientific, cat. no. 26183); rabbit anti-HA tag (C29F4) monoclonal antibody (1:1000 for Nav1.6 and 1:2000 for NF186; Cell Signaling, cat. no. 3724); mouse anti-ankyrin G antibody (1:50; Santa Cruz, cat. no. 12719); and mouse anti- $\beta$ III-tubulin antibody (1:1000; BioLegend, cat. no. 801202). The secondary antibodies were as follows: goat anti-rabbit AF555 (1:500; Thermo Fisher Scientific, cat. no. A21429); goat anti-rabbit AF647 Plus [1:500; AF(+)647; Thermo Fisher Scientific, cat. no. A32733], goat anti-mouse AF633 (1:500; Thermo Fisher Scientific, cat. no. A-21052); and goat anti-mouse AF(+)647 (1:500; Thermo Fisher Scientific, cat. no. A-21236).

\section{Cell culture}

Mouse neuroblastoma $x$ rat neuron hybrid ND7/23 cells (ECACC 92090903, Sigma Aldrich) were grown in high-glucose Dulbecco's Modified Eagle Medium (DMEM; Thermo Fisher Scientific, cat. no. 41965062) supplemented with $10 \%$ heat-inactivated fetal bovine serum (FBS; Thermo Fisher Scientific, cat. no. 10270106), 1\% penicillin-streptomycin (PS; Sigma Aldrich, cat. no. P0781), 1\% sodium pyruvate (Thermo Fisher Scientific, cat. no. 11360039), and 1\% L-glutamine (Thermo Fisher Scientific, cat. no. $25030024)$ at $37{ }^{\circ} \mathrm{C}$ and $5 \% \mathrm{CO}_{2}$. FBS was heat-inactivated via incubation at $56{ }^{\circ} \mathrm{C}$ for $30 \mathrm{~min}$. Mouse neuroblastoma N1E-115-1 cells (ECACC 08062511, Sigma Aldrich) were grown in high-glucose DMEM supplemented with $10 \% \mathrm{FBS}$ and $1 \% \mathrm{PS}$ at $37^{\circ} \mathrm{C}$ and $5 \% \mathrm{CO}_{2}$. For maintenance of the PiggyBAC $\mathrm{N} 1 \mathrm{E}-115-1^{\beta 1 \beta 2}$ stable cells, in addition to $10 \% \mathrm{FBS}$ and $1 \%$ PS we supplemented the medium with $3 \mu \mathrm{g} / \mathrm{ml}$ puromycin (Sigma Aldrich, cat. no. P8833). For the experiments that involved transfection and electrophysiological recordings, neuronal cells were used from passage 3-15 and were passaged three times a week.

For the microscopy experiments, neuroblastoma ND7/23 or N1E-115-1 cells were seeded on four-well Lab-Tek II chambered cover glasses (German \#1.5 borosilicate glass; Thermo Fisher Scientific, cat. no. 
155382) at the following densities: 50,000 ND7/23 cells per well for click labeling of NF186 and 100,000 ND7/23 cells per well, or 60,000 N1E-115-1 cells per well for click labeling of Nav1.6. Before cell seeding, chambered cover glasses were pre-coated with $10 \mu \mathrm{g} / \mathrm{ml}$ Poly-D-lysine (PDL; Sigma Aldrich, cat. no. P6407) solution in double-distilled water $\left(\mathrm{ddH}_{2} \mathrm{O}\right)$ for at least $4 \mathrm{~h}$ at room temperature (RT). The chambered cover glasses were washed three times with $\mathrm{ddH}_{2} \mathrm{O}$ and dried completely before cell seeding. For the electrophysiological recordings, 250,000 N1E-115-1 or $160,000-200,000$ PiggyBAC N1E-115-1 ${ }^{\beta 1 \beta 2}$ stable cells were seeded per well of a six-well plate a day before the transfection.

For the experiments that included genetic code expansion and click labeling of NF186 and Nav1.6, Gibco primary rat cortex neurons, from Sprague Dawley embryonic day 18 rats (Thermo Fisher Scientific, cat. no. A365512) were thawed and cultured based on the manufacturer's recommendation. The neurons were maintained in the B-27 ${ }^{\mathrm{TM}}$ Plus Neuronal Culture System (Thermo Fisher Scientific, cat. no. A3653401) that contained 2\% B27 Plus and 1\% PS. For the microscopy experiments, rat neurons were seeded in eight-well Lab-Tek II chambered cover glasses (German \#1.5 borosilicate glass; Thermo Fisher Scientific, cat. no. 155409) at a density of 100,000-110,000 neurons per well. Prior to the neuron seeding, chambered cover glasses were pre-coated with $20 \mu \mathrm{g} / \mathrm{ml}$ of PDL solution in $\mathrm{ddH}_{2} \mathrm{O}$ for $2 \mathrm{~h}$ at RT. Afterwards, they were washed three times with $\mathrm{ddH}_{2} \mathrm{O}$, dried completely, and pre-incubated with $250 \mu \mathrm{l}$ of a warm culture medium for at least $30 \mathrm{~min}$ at $37^{\circ} \mathrm{C}, 5 \% \mathrm{CO}_{2}$. Half of the culture medium was replaced every 3-4 days.

631 For click labeling of the LOF Nav1.6 variants, we used mouse hippocampal neurons. Our animal protocols 632 for the mouse hippocampal neuronal culture preparation were approved by the local Animal Care and 633 Use Committee (Regierungspraesidium Tübingen, Tübingen, Germany). Neurons were isolated from embryonic day $18 \mathrm{C} 57 \mathrm{BL} / 6 \mathrm{NCrl}$ mouse pups. The pregnant mice were cervically dislocated after asphyxiation with $\mathrm{CO}_{2}$, and then the embryos were taken out and decapitated immediately. The 636 hippocampi within the whole brain stored in cold magnesium- and calcium-free Hanks' Balanced Salt 637 Solution (HBSS; Thermo Fisher Scientific, cat. no. 14175053) were recognized under a dissecting 638 microscope (Olympus SZ 61, Shinjuku, Tokyo, Japan) and isolated using fine forceps. After the hippocampi 639 were washed three times with a cold HBSS solution, the hippocampal tissue was incubated for 14 min in $6402.5 \%$ trypsin (Thermo Fisher Scientific, cat. no. 15090046 ) at $37^{\circ} \mathrm{C}$ and then washed in DMEM with FBS 641 (PAN Biotech, cat. no. 3306-P131004) to block the enzyme digestion. Dissociated neurons were obtained 642 via gentle mechanical trituration and plated on four-well Lab-Tek II chambered cover glasses at a density of 120,000 neurons per well. The chambered glasses were pre-coated with $0.1 \mathrm{mg} / \mathrm{ml}$ of PDL solution in 
644

645

646

647

648

649

650

651

652

653

654

655

656

657

658

659

660

661

662

663

664

665

666

667

668

669

670

671

672

$\mathrm{ddH}_{2} \mathrm{O}$ prior to embryo preparation. After $6 \mathrm{~h}$, during which the neurons settled on the chambered coverslips in a $5 \% \mathrm{CO}_{2}$ humidified atmosphere at $37{ }^{\circ} \mathrm{C}$, the culture medium was replaced by the Neurobasal culture medium (Thermo Fisher Scientific, cat. no. 21103049) supplemented with B27 (Thermo Fisher Scientific, cat. no. 17504044), L-glutamine (Thermo Fisher Scientific, cat. no. 25030024), and PS (Thermo Fisher Scientific, cat. no. 15140122). Half of the neuronal culture medium was exchanged every 3-4 days.

\section{Generation of stable cell lines}

For the generation of N1E-115-1 $1^{\beta 1 \beta 2}$ stable cells, we used the PiggyBac Transposon system obtained from BioCat. Before we started the generation of the stable cell line, we determined that the appropriate puromycin concentration for the selection of stable clones was $3 \mu \mathrm{g} / \mathrm{ml}$. N1E-115-1 cells were seeded on a six-well plate at a density of 200,000 cells per well a day before the transfection. The following day, the cells were transfected with $0.5 \mu \mathrm{g}$ PiggyBAC $\mathrm{m} \beta 1$ plasmid, $0.5 \mu \mathrm{g}$ PiggyBAC $\mathrm{m} \beta 2$ plasmid, and $0.4 \mu \mathrm{g}$ Super PiggyBAC Transposase Expression vector (BioCat, cat. no. PB210PA-1-SBI). For the transfection, we used the JetPrime (Polyplus-transfection, cat. no. 114-15) reagent following the manufacturer's instructions $(2.8 \mu \mathrm{l}$ of JetPrime transfection reagent per $1.4 \mu \mathrm{g}$ of DNA). Cells were incubated with the transfection mix for $4.5 \mathrm{~h}$, and afterwards, medium was replaced. The day after the transfection, the selection was initiated by adding $3 \mu \mathrm{g} / \mathrm{ml}$ puromycin. To ensure that all the untransfected cells were eliminated, we increased the puromycin concentration for selection from $3 \mu \mathrm{g} / \mathrm{ml}$ to $6 \mu \mathrm{g} / \mathrm{ml}$ and added it to the cells a day after the transfection, in the evening. Seventy-two hours after the transfection, the cells were transferred from six-well plates to p10 Petri dishes (Greiner Bio-one, cat. no. 664160). The cells were further propagated in p10 Petri dishes and p20 Petri dishes (Greiner Bio-one, cat. no. 639160) until single cell clusters were formed. After the cell cluster formation, we selected single clones and propagated them further in p10 dishes. Stocks of various clones were frozen for further analysis. To confirm that $m S C N 1 B$ and $m S C N 2 B$ were stably incorporated into the genomes of the N1E-115-1 cells, we extracted genomic(g)DNA from different clones with the PureLink gDNA kit (Thermo Fisher, cat. no. K1820-01) and PCR-amplified $m S C N 1 B$ and $m S C N 2 B$ genes from the gDNA. The clones with correct patterns on the gel were used in the experiments. The primers used for amplification of gDNA are given in Supplementary

\section{Table 5.}

\section{Transfections}


673 For click labeling of NF186, ND7/23 cells were transfected a day after they were seeded with the 674 Lipofectamine 2000 transfection reagent (Thermo Fisher Scientific, cat. no. 11668027), as described in 675 detail (Arsić et al., 2022, Arsić and Nikić-Spiegel, 2022). Briefly, the cells were seeded into four-well Lab676 Tek II chambered cover glasses and transfected with a total DNA amount of $1 \mu \mathrm{g}$ per well (0.5 $\mu \mathrm{g}$ WT/TAG 677 plasmid and $0.5 \mu \mathrm{g}$ NES PyIRS/tRNA ${ }^{\text {Pyl }}$ plasmid) and a DNA/Lipofectamine 2000 reagent ratio of $6781 \mu \mathrm{g} / 2.4 \mu \mathrm{l}$. After the addition of the transfection mix, TCO*A-Lys/1M HEPES was added to the medium at 679 a final concentration of $250 \mu \mathrm{M}$. After $6 \mathrm{~h}$, the medium was replaced, TCO*A-Lys/1M HEPES was diluted in the same way as described above and added again to the medium, and the cells were incubated overnight. The following day, the cells were click-labeled and immunostained.

For click labeling of Nav1.6, ND7/23 or N1E-115-1 cells were transfected a day after they were seeded with Lipofectamine 3000 transfection reagent (Thermo Fisher, cat. no. L3000015). We used a DNA/Lipofectamine 3000 reagent ratio of $1 \mu \mathrm{g} / 1.5 \mu \mathrm{l}$ and a DNA/P3000 ratio of $1 \mu \mathrm{g} / 2 \mu \mathrm{l}$. For the microscopy experiments, ND7/23 or N1E-115-1 cells were seeded into four-well Lab-Tek II chambered cover glasses and transfected using a total of $1.8 \mu \mathrm{g}$ of DNA per well $(0.8 \mu \mathrm{g} \mathrm{Nav1.6} \mathrm{WT/TAG,}$ $0.8 \mu \mathrm{g}$ NES PyIRS/tRNA ${ }^{\text {Pyl }}, 0.1 \mu \mathrm{g}$ pACEmam2 $\mathrm{m} \beta 1$, and $0.1 \mu \mathrm{g}$ pMDC $\mathrm{m} \beta 2$ ). For the electrophysiological recording experiments, N1E-115-1 cells were seeded into six-well plates and transfected with a total amount of $8.4 \quad \mu \mathrm{g}$ DNA per well $\left(4 \mu \mathrm{g}\right.$ Nav1.6, $4 \mu \mathrm{g}$ NES PyIRS/tRNA ${ }^{\text {Pyl }}$, and $0.4 \mu \mathrm{g}$ pACEMam2_m $\beta 1$ _m $\beta 2$ _mGFP). N1E-115-1 ${ }^{\beta 1 \beta 2}$ cells were seeded into six-well plates and transfected with a total amount of $5 \mu \mathrm{g}$ of DNA per well [2.5 $\mu \mathrm{g}$ Nav1.6-P2A-eGFP and $2.5 \mu \mathrm{g}$ NES PyIRS/tRNA ${ }^{\text {Pyl }}$ (which was a gift from Dr. Edward Lemke)]. After the addition of the transfection mix, TCO*A-Lys/1 M HEPES was added to the medium at a final concentration of $250 \mu \mathrm{M}$. After $6 \mathrm{~h}$, the medium was replaced and TCO*A-Lys was diluted in the same way as described above and then added again to the medium. After 2 days of incubation at $37^{\circ} \mathrm{C}$ and $5 \% \mathrm{CO}_{2}$, the ND7/23 or N1E-115-1 cells were click-labeled for the microscopy experiments. For the whole-cell patch clamp recordings, the cells were assessed for a fluorescent signal, counted, and reseeded in $35 \mathrm{~mm}$ Petri dishes (Greiner Bio-one, 698 cat. no. 627160) at a density of around 180,000 cells per dish without further addition of 699 TCO*A-Lys/1 M HEPES. The cells were left at $37^{\circ} \mathrm{C}$ and $5 \% \mathrm{CO}_{2}$ for $2-4 \mathrm{~h}$ until the electrophysiological 700 recordings were performed. The cells were always recorded on the same day.

701 Primary Sprague Dawley rat cortical neurons were transfected with Lipofectamine 2000 as described 702 previously (Arsić et al., 2022, Arsić and Nikić-Spiegel, 2022) or with Lipofectamine 3000 following the 703 manufacturer's instructions. For click labeling of NF186, neurons were transfected with 
704

705

706

707

708

709

710

711

712

713

714

715

716

717

718

719

720

721

722

723

724

725

726

727

728

729

730

731

732

733

Lipofectamine 2000 on DIV 7; and for click labeling of Nav1.6, neurons were transfected with Lipofectamine 2000 or 3000 on DIV 8. Neurons were seeded into eight-well Lab-Tek II chambered cover glasses, and a total amount of $1 \mu \mathrm{g}$ per well was used for the transfection with Lipofectamine 2000 (0.5 $\mu \mathrm{g}$ Nav1.6 WT/TAG or NF186 WT/TAG and $0.5 \mu \mathrm{g}$ NES PyIRS/tRNA ${ }^{\text {Pyl }}$ ). For the transfection with Lipofectamine 3000 , we used a total amount of $0.5 \mu \mathrm{g}$ per well (at the DNA/Lipofectamine 3000 ratio of $1 \mu \mathrm{g} / 1.5 \mu \mathrm{l}$ and the DNA/P3000 ratio of $1 \mu \mathrm{g} / 2 \mu \mathrm{l})$. The transfection mix was prepared in $25 \mu \mathrm{l}$ Opti-MEM Reduced serum media (Thermo Fisher, cat. no. 31985062) without the addition of antibiotics. Then $250 \mu l$ of the medium was removed and saved for later at $37^{\circ} \mathrm{C}$ and $5 \% \mathrm{CO}_{2}$ (conditioned medium). The entire transfection mix was added to the well that contained the remaining $250 \mu$ of the medium. The neurons that were transfected with Lipofectamine $2000 / 3000$ were incubated with the transfection mix for at least $6 \mathrm{~h}$. Afterwards, the transfection mix was removed, $250 \mu \mathrm{l}$ of the previously collected conditioned medium was added, and $250 \mu \mathrm{l}$ of the fresh culture medium was added to each well. TCO*A-Lys/1 M HEPES was added to a final concentration of $250 \mu \mathrm{M}$, and the cells were incubated for 3 days at $37^{\circ} \mathrm{C}$ and $5 \% \mathrm{CO}_{2}$. After 3 days of incubation, the medium that contained TCO*A-Lys was completely removed and replaced with one-half of the conditioned medium and one-half of the fresh culturing medium. In the controls that did not contain TCO*A-Lys/1 M HEPES, one-half of the medium was replaced with the fresh culture medium. Click labeling was performed on the following day. For localization of the LOF Nav1.6 variants, mouse hippocampal neurons were transfected with Lipofectamine 2000 as described previously on DIV 78. The only difference was that the neurons were seeded in four-well Lab-Tek II chambered cover glasses. Therefore, the amounts of the DNA and the Lipofectamine 2000 transfection reagent were scaled up to correspond to the size of the four-well Lab-Tek II chambered cover glasses (two times more DNA and Lipofectamine 2000 were used).

\section{Click labeling of NF186 and Nav1.6}

Click labeling of ND7/23 cells expressing NF186-HA was performed a day after the transfection. Until the labeling, the cells were incubated with TCO*A-Lys. The cells were labeled with $1.5 \mu \mathrm{M}$ ATTO488-tz diluted in a warm culture medium at $37^{\circ} \mathrm{C}$ and $5 \% \mathrm{CO}_{2}$. After $10 \mathrm{~min}$ of incubation, the dye solution was removed, the cells were washed 1-2 times with $0.01 \mathrm{M}$ phosphate-buffered saline (PBS; $137 \mathrm{mM} \mathrm{NaCl}, 10 \mathrm{mM}$ $\mathrm{Na}_{2} \mathrm{HPO}_{4}, 1.8 \mathrm{mM} \mathrm{KH}_{2} \mathrm{PO}_{4}, 2.7 \mathrm{mM} \mathrm{KCl}, \mathrm{pH}$ 7.4), fixed with 4\% paraformaldehyde (PFA; Sigma-Aldrich, cat. no. 158127) in $0.1 \mathrm{M}$ phosphate buffer (PB) for $15 \mathrm{~min}$ at RT and washed 3 times with PBS. Click labeling

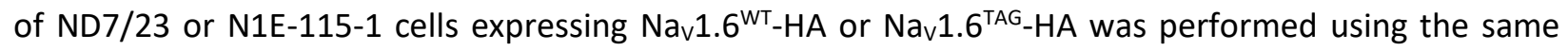


734

735

736

737

738

739

740

741

742

743

744

745

746

747

748

749

750

751

752

753

754

755

756

757

758

759

760

761

762

763

procedure described previously except that click labeling was performed $40-46 \mathrm{~h}$ after the transfection with $3 \mu \mathrm{M}$ ATTO488-tz.

Click labeling of living rat cortical neurons or mouse hippocampal neurons was performed 4 days after the transfection except for the experiments that involved dSTORM, where rat neurons were click-labeled 4-6 days after the transfection. The medium that contained TCO*A-Lys was removed a day before the click labeling. For the click labeling of NF186-HA, rat neurons were labeled with $5 \mu \mathrm{M}$ ATTO488-tz diluted in a warm culture medium for $10 \mathrm{~min}$ at $37^{\circ} \mathrm{C}$ and $5 \% \mathrm{CO}_{2}$. After $10 \mathrm{~min}$ of incubation with the dye, the medium was removed and the neurons were fixed with 4\% electron-microscopy-grade PFA (Electron Microscopy Sciences, cat. no. 15710) diluted in a cytoskeleton-protective buffer (PEM; 80 mM PIPES, 5 mM EGTA, 2 $\mathrm{mM} \mathrm{MgCl} 2 ; \mathrm{pH} \mathrm{6.8)} \mathrm{for} 15$ min at RT and washed 3 times in PBS (5 min per wash). For the live-cell imaging of NF186, after the click labeling, the cells were washed 2-3 times with the culture medium, placed in a Hibernate E medium that contained 2\% B27 Plus and 1\% PS, and kept in the incubator until the imaging $\left(37^{\circ} \mathrm{C}, 5 \% \mathrm{CO}_{2}\right)$. Click labeling of the rat cortical neurons or mouse hippocampal neurons expressing Nav1.6-HA was performed with the same procedure, except that before the click labeling, the neurons were washed 3 times with Tyrode's solution $(100 \mathrm{mM} \mathrm{NaCl}, 5 \mathrm{mM} \mathrm{KCl}, 5 \mathrm{mM} \mathrm{MgCl}$, $2 \mathrm{mM} \mathrm{CaCl}, 15 \mathrm{mM}$ D-glucose, 10 mM HEPES; pH 7.4, osmolarity 243-247 mOsm) and incubated for 3 min in 1\% BSA/Tyrode's solution (Bessa-Neto et al., 2021). After 10 min of incubation with the ATTO488-tz dye, the neurons were washed 4 times with Tyrode's solution, fixed immediately, and washed 3 times in PBS (5 min per wash). For the live-cell imaging of Nav1.6, upon the click labeling, the rat neurons were placed in the Hibernate E medium that contained $2 \% \mathrm{~B} 27$ Plus and $1 \%$ PS and kept in the incubator until the imaging $\left(37^{\circ} \mathrm{C}\right.$, $\left.5 \% \mathrm{CO}_{2}\right)$.

\section{Immunostaining}

For immunostaining of neuroblastoma cells and primary neurons, all the blocking steps were performed at RT for $1 \mathrm{~h}$. Incubation with primary antibodies was performed $\mathrm{ON}$ at $4^{\circ} \mathrm{C}$ while incubation with secondary antibodies was performed at RT for $1 \mathrm{~h}$. After the incubation with the primary and secondary antibodies, the cells were washed 3 times with PBS ( 5 min per wash). Then they were kept in PBS at $4^{\circ} \mathrm{C}$ until the imaging.

For immunostaining of ND7/23 cells expressing NF186-HA, the cells were permeabilized with $0.2 \%$ Tween 20/PBS for 30 min. Afterwards, the cells were blocked with 5\% FBS. Mouse anti-HA primary and goat anti-mouse $\mathrm{AF}(+) 647$ secondary antibodies were diluted in a blocking buffer. For immunostaining 
764

765

766

767

768

769

770

771

772

773

774

775

776

777

778

779

780

781

782

783

784

785

786

787

788

789

790

791

792

793

of rat neurons expressing NF186-HA, the neurons were permeabilized and blocked in a buffer that contained $0.2 \%$ Triton X-100 (Sigma-Aldrich, cat. no. X100), 10\% goat serum (GS; Thermo Fisher, cat. no. 16210072), and 3\% BSA in PBS. Rabbit anti-HA and mouse-anti ankG primary antibodies were diluted in the blocking buffer. Goat anti-rabbit AF555 and goat-anti mouse AF633 secondary antibodies were diluted in $3 \%$ BSA/PBS.

For immunostaining of Nav1.6-HA, neuroblastoma cells or primary neurons were permeabilized with $0.1 \%$ Triton X-100/PBS for 10 min and blocked in a buffer that contained $10 \%$ GS and 3\% BSA. Rabbit anti-HA primary antibody was diluted in the blocking buffer. Goat anti-rabbit AF555 secondary antibody was diluted in 3\% BSA/PBS. For the AIS quantification of the rat cortical neurons expressing Nav1.6-HA, in addition to the rabbit anti-HA primary antibody and the goat-anti rabbit AF555 secondary antibody, neurons were immunostained with mouse anti-ankG primary antibody and goat anti-mouse AF633 secondary antibody.

For dSTORM imaging of Nav1.6, rat cortical neurons were immunostained with rabbit anti-HA antibody as described above, except that instead of goat-anti rabbit AF555, goat-anti rabbit $A F(+) 647$ secondary antibody was used.

\section{Adeno-associated viruses (AAVs) production, purification and titration}

A fluorescent reporter and genetic code expansion components-nuclear localization sequence (NLS)-mCherry-GFP ${ }^{\mathrm{Y} 39 \mathrm{TAG}}$, NES PyIRS ${ }^{\mathrm{AF}}$, one copy tRNA ${ }^{\mathrm{Pyl}}$, and eRF1 ${ }^{\mathrm{E5} 5 \mathrm{D}}$ [gifts from Dr. Edward Lemke (EMBL, Heidelberg, and IMB, Mainz)], and a cassette containing four copies of tRNA ${ }^{\text {Pyl }}$ [a gift from Jason Chin (MRC Laboratory of Molecular Biology, Cambridge, UK)]-were separately cloned into a self-complementary AAV vector plasmid (encoding transgenes flanked by inverted terminal repeats) backbone (Supplementary Fig. 8a). AAV vectors were produced via triple-transfection of the HEK293T cells with the AAV vector plasmid, the AAV helper plasmid (encoding the AAV rep and cap genes; AAV7A2 and AAV9A2 caps were used in this study) and adenoviral helper plasmid at a 1:1:1 molar ratio using a polyethylenimine (PEI MAX; Polysciences, Warrington, USA, cat.no. 24765-1) as the transfection reagent. Ten $15 \mathrm{~cm}$ dishes were used to produce each AAV vector. In brief, 4 million HEK293T cells per dish were seeded 2 days before the transfection. A DNA mix (44 $\mu$ g plasmids in $790 \mu \mathrm{H}_{2} \mathrm{O}$ and $790 \mu \mathrm{l}$ of $300 \mathrm{mM} \mathrm{NaCl}$ per dish) and a PEI mix ( $352 \mu \mathrm{l} \mathrm{PEI}, 438 \mu \mathrm{l} \mathrm{H} \mathrm{H}_{2}$, and $790 \mu \mathrm{l}$ of $300 \mathrm{mM} \mathrm{NaCl}$ per dish) were mixed together, vortexed thoroughly, incubated at RT for $10 \mathrm{~min}$, and added to the cells dropwise. Three days after the transfection, the cells were harvested with a cell scraper and centrifuged at $800 \mathrm{~g}$ for $15 \mathrm{~min}$. The cell pellets were 
resuspended in a $5 \mathrm{ml}$ Benzonase buffer $(150 \mathrm{mM} \mathrm{NaCl}, 50 \mathrm{mM}$ Tris- $\mathrm{HCl}, 2 \mathrm{mM} \mathrm{MgCl} ; \mathrm{pH} 8.5)$ and lysed via 5 freeze-thaw cycles. Lysates were sonicated for $1 \mathrm{~min}$ and $20 \mathrm{sec}$ and incubated with $75 \mathrm{U} / \mathrm{mL}$ Benzonase (Merck, Darmstadt, Germany, cat. no. 1.01695.0001) at $37^{\circ} \mathrm{C}$ for $1 \mathrm{~h}$. Then the lysates were centrifuged at $4000 \mathrm{~g}$ for $15 \mathrm{~min} 2$ times to get rid of the cell debris.

The AAV vectors in the abovementioned supernatant were purified with an iodixanol (OptiPrep ${ }^{\mathrm{TM}}$ (Iodixanol); Progen, Heidelberg, Germany, cat. no. 1114542) gradient. Each vector was loaded into ultracentrifugation tubes (Seton Scientific, Petulama, CA, USA) through a Pasteur pipette, followed by $2 \mathrm{ml}$ of $15 \%, 25 \%, 40 \%$, and $60 \%$ iodixanol solution. The tubes were sealed and centrifuged at $50,000 \mathrm{rpm}$ at $4^{\circ} \mathrm{C}$ for $2 \mathrm{~h}$ in an OptimaTM L-90K ultracentrifuge using the 70.1Ti rotor (Beckman Coulter, Brea, CA, USA). After the centrifugation, the $1 \mathrm{ml}$ solution at the interface of $40 \%$ and $60 \%$ phases was collected and stored at $-80^{\circ} \mathrm{C}$.

The AAV titers were measured with droplet digital PCR (ddPCR). The AAV samples were diluted at 1: $10^{6}$. Reaction solutions $(20 \mu \mathrm{l})$ that contained $900 \mathrm{nM}$ of each primer, $25 \mathrm{nM}$ of the probe, $10 \mu \mathrm{l}$ of the 2x ddPCR Supermix for probes (no dUTP; Bio-Rad, Hercules, USA, cat. no. 1863024), and $5 \mu$ l of the diluted AAV sample were prepared for the droplet generation. The droplets were generated with a QX200 Droplet Generator (Bio-Rad, Hercules, USA), and then transferred to a 96-well PCR plate. PCR was run in a C1000 Touch Thermal Cycler (Bio-Rad, Hercules, USA), and the results were read by QX200 Droplet Reader (Bio-Rad, Hercules, USA). The primers used for ddPCR are given in Supplementary Table 5.

\section{Transduction with AAVs and transfections of primary neurons}

For the transduction of the primary neurons with $A A V s$, we adjusted the desired the multiplicity of infection (MOI) by diluting AAV stocks in a warm fresh culture medium supplemented with $2 \%$ B27 Plus and $1 \%$ PS. Before the transduction, an equal amount of conditioned medium was added to the prewarmed fresh medium that contained the AAV.

Primary neurons were transduced at DIV 5 with AAV9A2 or AAV7A2 encoding the NLS-mCherry-GFP ${ }^{\text {Y39TAG }}$ expressed from the CMV promoter without the addition of TCO*A-Lys. We used an MOI of 50,000. For the genetic code expansion of GFP ${ }^{\mathrm{Y} 9 T A G}$, we co-transduced rat primary cortex neurons at DIV 8 with AAV9A2 vectors encoding the CMV-NLS-mCherry-GFP ${ }^{\text {Y39TAG }}$ (AAV\#6) and the following genetic code expansion components: (a) CMV-NES PyIRS ${ }^{A F}$ (AAV\#1) and four copies of tRNA ${ }^{\text {Pyl }}$ (AAV\#2); (b) CMV-NES PyIRS ${ }^{A F}$ (AAV\#1), 4xtRNA ${ }^{\text {Pyl }}$ (AAV\#2), and mutant eukaryotic release factor eRF1 ${ }^{\text {E55D }}$ expressed from the CMV promoter (AAV\#3); (c) CMV-NES PyIRS ${ }^{A F}$ (AAV\#1) and AAV9A2 carrying minimal 
824 (min)CMV-eRF1 ${ }^{\text {E55D }}$ and one copy of tRNA ${ }^{\text {Pyl }}$ (AAV\#5); (d) AAV9A2 carrying minCMV-NES PyIRS ${ }^{A F}$ (AAV\#4) 825 and one copy of tRNA ${ }^{\text {Pyl }}$ and AAV9A2 carrying mineRF1 ${ }^{\text {E55D }}$ and one copy of tRNA ${ }^{\text {Pyl }}$ (AAV\#5; Supplementary Fig. 8a). An MOI of 5,000 was used for each AAV. TCO*A-Lys/1 M HEPES was added 827 immediately upon transduction at a final concentration of $250 \mu \mathrm{M}$. The neurons were assessed daily for 828 their fluorescent signal and viability. Three days after the transductions, the neurons were fixed with the

$8294 \%$ PFA/PEM buffer for 15 min at RT, washed 3 times in PBS (5 min per wash), and left at $4^{\circ} \mathrm{C}$ until the 830 imaging.

831 For click labeling of Nav1.6, we transfected primary rat cortical neurons at DIV 8 with $0.5 \mu \mathrm{g} \mathrm{Nav} 1.6^{\mathrm{TAG}}-\mathrm{HA}^{\mathrm{A}}$ 832 using Lipofectamine 2000, as described in the transfection section. After $6 \mathrm{~h}$, we removed the transfection mix and added $250 \mu \mathrm{l}$ of the condition medium that was saved previously and $250 \mu \mathrm{l}$ of the fresh medium that contained the same combinations of AAV92A used for the NLS-mCherry-GFP ${ }^{\text {Y39TAG }}$. For each AAV, we used an MOI of 15,000. TCO*A-Lys/1 M HEPES was added immediately upon the transduction at a final concentration of $250 \mu \mathrm{M}$. The neurons were assessed daily for viability. Three days after the transfection and transduction, we click-labeled the neurons with $5 \mu \mathrm{M}$ ATTO488-tz, as described in the Click labeling section, and fixed neurons immediately with the 4\% PFA/PEM buffer for $15 \mathrm{~min}$ at RT. The neurons were washed 3 times in PBS (5 min per wash) and immunostained afterwards, as described in the Immunostaining section.

\section{Fixed- and live-cell imaging}

842 Confocal imaging was performed on an LSM 710 confocal scanning microscope (Carl Zeiss, Oberkochen, 843 Germany) equipped with the following laser lines (nm): 405, 440, 458, 488, 514, 561, and 633. Images 844 were acquired with an oil Plan-Apochromat 63x objective (NA 1.4) and with the following settings: a 8451024 x 1024 pixel frame size, a 16-bit image depth, $2 \times$ line averaging, a $6.30 \mu$ s pixel dwell time, and 0.132 $846 \mu \mathrm{m}$ pixel size. We used a $488 \mathrm{~nm}$ laser line to excite ATTO488-tz, a $561 \mathrm{~nm}$ laser line to excite AF555, and 847 a $633 \mathrm{~nm}$ laser line to excite $\mathrm{AF}(+) 647$ or AF633. The pinhole was set at 1 Airy Unit in all the channels, and 848 the emission light was collected sequentially. Images were acquired in two channels (488 nm and $561 \mathrm{~nm}$, 849 or $488 \mathrm{~nm}$, and $633 \mathrm{~nm}$ ) or three channels ( $488 \mathrm{~nm}, 561 \mathrm{~nm}$ and $633 \mathrm{~nm}$ ) channels, as single planes or as $850 \quad$ Z-stacks with a $0.42 \mu \mathrm{m}$ step size.

851 For live-cell confocal imaging, we used a temperature module and a heating insert (PeCon, Erbach 852 Germany) that was warmed up to $37^{\circ} \mathrm{C}$. Live rat cortical neurons were imaged in the Hibernate $\mathrm{E}$ medium 853 supplemented with 2\% B27 Plus and 1\% PS. 
854

855

856

857

858

859

860

861

862

863

864

865

866

867

868

869

870

871

872

873

874

875

876

877

878

879

880

881

882

Widefield epifluorescence and 3D dSTORM imaging were performed on an Inverted Nikon Eclipse Ti2-E (Nikon Instruments) equipped with an XY motorized stage; a Perfect Focus System; oil-immersion objectives (Apo 60x, NA 1.4, oil and HP Apo TIRF 100xH, NA 1.49, and Oil); an N-STORM module; the following filter cubes: 488 (AHF; EX 482/18; DM R488; BA 525/45), 561 (AHF; EX 561/14; DM R561; BA 609/54), and 647 (AHF; EX 628/40; DM660; BA 692/40); Nikon Normal STORM (T660lpxr, ET705/72 m); the following laser lines (nm): 405, 488, 561 and 647; and ORCA-Flash 4.0 sCMOS camera (Hamamatsu Photonics). The setup was controlled by NIS-Elements AR software (Nikon Instruments).

Images of rat cortical neurons expressing NF186 ${ }^{\mathrm{WT}}$-HA were acquired in the widefield mode with an Apo $60 \times$ oil objective, 30 ms exposure time, $1024 \times 1024$ pixel frame size, $16-$ bit image depth, and pixel size $0.27 \mu \mathrm{m}$. For the widefield imaging, a fluorescent lamp (Lumencor Sola SE II) was used as a light source, with the excitation light intensity set at $20 \%$.

\section{Image processing}

All the images shown in the main and supplementary figures, except for the dSTORM images, were processed in ImageJ/Fiji software (Schindelin et al., 2012). Raw confocal single planes or Z-stacks and widefield single planes images were imported in ImageJ/Fiji. The brightness and contrast of the 16-bit images were linearly adjusted. The Z-stacks were converted into maximum intensity projections prior to the linear adjustment of the brightness and contrast. For presentation purposes, the all images were converted to an 8-bit depth, exported as .tiff files, and arranged into figures using Adobe Illustrator.

The schemes presented in the manuscript were made in BioRender.com.

\section{D dSTORM imaging and image processing}

3D dSTORM imaging was performed on the N-STORM module of the Inverted Nikon Eclipse Ti2-E microscope. 3D dSTORM images of the rat cortical neurons expressing Nav1.6 were acquired with an HP Apo TIRF 100×H objective, a $647 \mathrm{~nm}$ laser line (LU-NV Series Laser Unit), and a Nikon Normal STORM filter cube. The emitted light was imaged with an ORCA-Flash 4.0 sCMOS camera that contained a cylindrical lens introduced in the light path (Huang et al., 2008). Prior to the 3D dSTORM imaging, the AIS of interest was assessed for click labeling. Afterwards, an image of the neuron expressing Nav1.6-HA was captured with a $647 \mathrm{~nm}$ laser (HA channel) set at 1\% of the power. Afterwards, the same neuron was imaged with 3D dSTORM. The 3D dSTORM imaging was performed in the total internal reflection fluorescence (TIRF) or highly inclined and laminated sheet microscopy (HILO) mode with continuous $647 \mathrm{~nm}$ laser illumination set at full power (100\%). The frame size was $128 \times 128$ pixels, and the image depth, 16 -bit. For each 3D 
dSTORM image, 20,000-30,000 frames were acquired at 33,3 Hz. During the acquisition, $405 \mathrm{~nm}$ laser illumination set at 5\% was used. The 3D dSTORM was calibrated previously using TetraSpeck Microspheres (Thermo Fisher, cat. no. T7279) following NIS-Elements' instructions. The 3D dSTORM imaging was performed in the GLOX BME buffer. GLOX BME buffer, which was prepared fresh prior to use by mixing $7 \mu \mathrm{l} \mathrm{BME}, 7 \mu \mathrm{l}$ GLOX solution [14 mg glucose oxidase (Sigma Aldrich, cat. no. G2133)], $50 \mu$ l catalase (17 mg/ml; Sigma Aldrich, cat. no. C3155), and $200 \mu$ l Buffer A (10 mM Tris; pH 8, 50 mM NaCl) with $690 \mu l$ Buffer B (50 mM Tris,pH 8, $10 \mathrm{mM} \mathrm{NaCl}$ ) that contained 20\% w/v glucose (Sigma Aldrich, cat. no. D9559). The buffer was prepared on ice.

The 3D dSTORM images were processed in NIS-Elements AR software. The molecule identification settings were set at the defaults for the 3D dSTORM analysis: a minimum width of $200 \mathrm{~nm}$, a maximum width of $700 \mathrm{~nm}$, an initial fit width of $300 \mathrm{~nm}$, a maximum axial ratio of 2.5, and a maximum displacement of 1 . The minimum height for the peak detection was set at 100 , and the localization analysis was performed with overlapping peak algorithms and drift correction. The 3D dSTORM images were reconstructed with a Gaussian rendering size of $10 \mathrm{~nm}$ in NIS Elements AR, and the final 3D dSTORM images (with z position height maps) were exported as .tiff files. The $Z$ rejected molecules were excluded from the final images. The .tiff images were imported and arranged into figures using Adobe Illustrator. Scale bars for dSTORM images and corresponding TIRF images were added in NIS-Elements AR software. Scale bars for all the other (widefield or confocal images) were added in ImageJ/Fiji.

\section{AIS quantification}

For the quantitative measurements of the AIS length of the rat primary neurons expressing NF186 or Nav1.6, we used the previously described MATLAB ais.m script (Grubb, 2021, Grubb and Burrone, 2010). Images were acquired on an LSM 710 confocal scanning microscope, as described in the fixed- and live-cell imaging section. We processed the images as described in the instructions of the ais.m script. Briefly, the confocal images used for the quantitative measurements (shown in Fig. $2 \mathrm{~d}$ and Fig. 3f) of the AIS length in the neurons expressing NF186 and Nav1.6 immunostained with anti ankG were processed in ImageJ/Fiji software. Raw confocal single planes or Z-stacks were imported in ImageJ/Fiji, color channels were split, the brightness and contrast of the 16-bit images were linearly adjusted, and different color channels were exported as separate 16-bit .tiff files. The Z-stacks were converted into maximum intensity projections prior to the linear adjustment of the brightness and contrast. The processed images were imported in MATLAB and analyzed with ais.m script (Grubb, 2021, Grubb and Burrone, 2010). The AIS lengths of the 
neurons expressing recombinant NF186 or Nav1.6 (measured in the ankG channel) were calculated automatically in MATLAB using the ais.m script.

The AIS fluorescence intensity of the click-labeled mouse primary hippocampal neurons expressing WT or LOF Nav1.6 variants (shown in Fig. 5d) were measured in ImageJ/Fiji with a previously described macros set (https://github.com/cleterrier/Measure_ROIs). Images were acquired on an LSM 710 confocal scanning microscope, as described in the fixed-and live-cell imaging section. We processed images in ImageJ/Fiji using a previously described macros set (https://github.com/cleterrier/Process_Images) and measured the fluorescence intensity along the AIS in the click channel. Briefly, the processed images were imported in ImageJ/Fiji, and the line tracings along the AIS were generated semi-automatically using the NeuronJ plugin (Meijering et al., 2004), converted into ImageJ/Fiji regions of interest (ROIs), and saved as a .zip files. Afterwards, raw 16-bit images were imported in ImageJ/Fiji together with their corresponding previously generated ROIs, the fluorescence intensity was automatically measured in the click channel, and the results were exported as .csv files. For the statistical analysis, we used a mean intensity measured along the ROI (along the AIS line tracing) corrected by subtracting the background (the "Corr Mean"). The line width of the ROI for each AIS was individually adjusted according to the width of the AIS.

All the image analyses were performed blind per experimental group. We did not image/analyze neurons overexpressing NF186 that had a clearly mislocalized HA signal present in all the processes. Due to the lower expression level of the recombinant Nav1.6, we did not observe any neurons overexpressing Nav1.6. Therefore, we imaged all the transfected cells showing click labeling.

\section{Electrophysiological recordings}

Standard whole-cell voltage clamp recordings were performed in ND7/23, N1E-115-1, or N1E-115-1 $11 \beta 2$ cells using an Axopatch 200B amplifier, a Digitata 1440A digitizer, and Clampex 10.2 data acquisition software (Axon Instruments, Union City, CA, USA) in the presence of $500 \mathrm{nM}$ tetrodotoxin (TTX). The cells were held at $-100 \mathrm{mV}$. Currents were filtered at $5 \mathrm{kHz}$ and digitized at $20 \mathrm{kHz}$. Borosilicate glass pipettes had a final tip resistance of 1.8-2.5 $\mathrm{M} \Omega$ when filled with the internal recording solution. The pipette solution contained (in mM) $10 \mathrm{NaCl}, 1 \mathrm{EGTA}, 10 \mathrm{HEPES}$, and $140 \mathrm{CsF}$. The $\mathrm{pH}$ was adjusted to 7.3 with $\mathrm{CsOH}$, and the osmolarity was adjusted to $290 \mathrm{mOsm} / \mathrm{kg}$ with mannitol. The bath solution contained (in $\mathrm{mM}) 140 \mathrm{NaCl}, 3 \mathrm{KCl}, 1 \mathrm{MgCl}_{2}, 1 \mathrm{CaCl}_{2}, 10 \mathrm{HEPES}, 20 \mathrm{TEACl}$ (tetraethylammonium chloride), $5 \mathrm{CsCl}$, and $0.1 \mathrm{CdCl}_{2}$. The $\mathrm{pH}$ was adjusted to 7.3 with $\mathrm{CsOH}$, and the osmolarity, to $320 \mathrm{mOsm} / \mathrm{kg}$ with mannitol. 
943

944

945

946

947

948

949

950

951

952

953

954

955

956

957

958

959

960

961

962

963

964

The electrophysiology data were analyzed as previously described (Liu et al., 2019). Briefly, the activation curve (conductance-voltage relationship) was derived from the current-voltage relationship. The latter was obtained by measuring the peak current at various step depolarizations from the holding potential of $-100 \mathrm{mV}$ according to the following formula:

$$
g(V)=\frac{I}{V-V_{\text {rev }}}
$$

Note that g represents the conductance, I represents the recorded peak current at the test potential (V) while $\mathrm{V}_{\text {rev }}$ represents the apparent observed $\mathrm{Na}^{+}$reversal potential.

A standard Boltzmann function was fit to the activation curves according to the following formula:

$$
g(V)=\frac{g_{\max }}{1+\exp \left[\left(V-V_{1 / 2}\right) / k_{v}\right]}
$$

Note that g represents the conductance, I represents the recorded current amplitude at the test potential (V), $\mathrm{V}_{\text {rev }}$ represents the $\mathrm{Na}^{+}$reversal potential, $\mathrm{g}_{\max }$ represents the maximal conductance, $\mathrm{V}_{1 / 2}$ represents the voltage of a half-maximal activation while kv represents a slope factor.

Steady-state inactivation was determined using 100 ms conditioning pulses to various potentials followed by the test pulse to $-10 \mathrm{mV}$ at which the peak current reflected the percentage of non-inactivated channels. A standard Boltzmann function was fit to the inactivation curves according to the following formula:

$$
I(V)=\frac{I_{\max }}{1+\exp \left[\left(V-V_{1 / 2}\right) / k_{v}\right]}
$$

Note that I represents the recorded current amplitude at the conditioning potential (V), I $I_{\max }$ represents the maximal current amplitude, $\mathrm{V}_{1 / 2}$ represents the voltage of half-maximal inactivation while $\mathrm{k}_{\mathrm{v}}$ represents a slope factor.

All the data were analyzed using the Clampfit software of pClamp 10.6 (Axon Instruments), Microsoft Excel (Microsoft Corporation, Redmond, WA, USA), or Igor Pro (Wavemetrics, Portland, OR, USA).

\section{Statistical analyses}

Statistical analyses (Shapiro-Wilk normality test, Levene's test for equality of variances, one-way ANOVA, and Kruskal-Wallis non-parametric Hypothesis test) for the quantification of the AIS length and the quantification of the AIS fluorescence intensity were performed with IBM SPSS Statistics 28.01.0 (Armonk, 
New York, USA). A Shapiro-Wilk normality test indicated whether the data followed a normal distribution. The data that followed normal distribution were further tested for the homogeneity of variances. The data that met both assumptions (normality and homogeneity of variances), and did not have any significant outliers were further analyzed with one-way ANOVA with a Tukey posthoc. The data that followed normal distribution, but did not meet the assumption of the homogeneity of variance and/or had significant outliers were analyzed with non-parametric Kruskal-Wallis test with Dunn-Bonferroni posthoc (if required). The details are provided in Supplementary Tables 1, 2 and 4.

The statistical analyses of the electrophysiological recordings were performed using GraphPad Prism (GraphPad Software, Inc., San Diego, CA, USA). D’Agostino \& Pearson normality tests indicated whether the data followed a normal distribution. To compare the groups, the unpaired T-test was used for the data that followed a normal distribution while the non-parametric Mann-Whitney $U$ test was used for the data that did not follow normal distribution. To compare three groups of data, ANOVA on ranks with Dunnett's posthoc test was used for data that did not follow normal distribution. For all the statistical tests, the significance levels compared to the controls are indicated in the Supplementary Table $\mathbf{3}$ using the following symbols: $* p<0.05,{ }^{* *} p<0.01$, and ${ }^{* * *} p<0.001$. The corresponding electrophysiology graphs were created in IgorPro (Wavemetrics, Portland, OR, USA).

\section{References}

AKIN, E. J., HIGERD, G. P., MIS, M. A., TANAKA, B. S., ADI, T., LIU, S., DIB-HAJJ, F. B., WAXMAN, S. G. \& DIBHAJJ, S. D. 2019. Building sensory axons: Delivery and distribution of NaV1.7 channels and effects of inflammatory mediators. Sci Adv, 5, eaax4755.

AKIN, E. J., SOLE, L., DIB-HAJJ, S. D., WAXMAN, S. G. \& TAMKUN, M. M. 2015. Preferential targeting of Nav1.6 voltage-gated $\mathrm{Na}+$ Channels to the axon initial segment during development. PLoS One, 10, e0124397.

AKIN, E. J., SOLE, L., JOHNSON, B., BEHEIRY, M. E., MASSON, J. B., KRAPF, D. \& TAMKUN, M. M. 2016. Single-Molecule Imaging of Nav1.6 on the Surface of Hippocampal Neurons Reveals Somatic Nanoclusters. Biophys J, 111, 1235-1247.

ARSIĆ, A., HAGEMANN, C., STAJKOVIĆ, N., SCHUBERT, T. \& NIKIĆ-SPIEGEL, I. 2022. Minimal genetically encoded tags for fluorescent protein labeling in living neurons. Nature Communications, 13, 314.

ARSIĆ, A. \& NIKIĆ-SPIEGEL, I. 2022. Bioorthogonal click chemistry-based labelling of proteins in living neuronal cell lines and primary neurons. Protocol Exchange.

BARTOSCHEK, M. D., UGUR, E., NGUYEN, T. A., RODSCHINKA, G., WIERER, M., LANG, K. \& BULTMANN, S. 2021. Identification of permissive amber suppression sites for efficient non-canonical amino acid incorporation in mammalian cells. Nucleic Acids Res.

BESSA-NETO, D., BELIU, G., KUHLEMANN, A., PECORARO, V., DOOSE, S., RETAILlEAU, N., CHEVRIER, N., PERRAIS, D., SAUER, M. \& CHOQUET, D. 2021. Bioorthogonal labeling of transmembrane proteins with non-canonical amino acids unveils masked epitopes in live neurons. Nat Commun, 12, 6715. 
1005

1006

1007

1008

1009

1010

1011

1012

1013

1014

1015

1016

1017

1018

1019

1020

1021

1022

1023

1024

1025

1026

1027

1028

1029

1030

1031

1032

1033

1034

1035

1036

1037

1038

1039

1040

1041

1042

1043

1044

1045

1046

1047

1048

1049

1050

1051

BIENERT, S., WATERHOUSE, A., DE BEER, T. A., TAURIELLO, G., STUDER, G., BORDOLI, L. \& SCHWEDE, T. 2017. The SWISS-MODEL Repository-new features and functionality. Nucleic Acids Res, 45, D313D319.

BORNER, K., KIENLE, E., HUANG, L. Y., WEINMANN, J., SACHER, A., BAYER, P., STULLEIN, C., FAKHIRI, J., ZIMMERMANN, L., WESTHAUS, A., BENEKE, J., BEIL, N., WIEDTKE, E., SCHMELAS, C., MILTNER, D., RAU, A., ERFLE, H., KRAUSSLICH, H. G., MULLER, M., AGBANDJE-MCKENNA, M. \& GRIMM, D. 2020. Pre-arrayed Pan-AAV Peptide Display Libraries for Rapid Single-Round Screening. Mol Ther, 28, 1016-1032.

CATTERALL, W. A., GOLDIN, A. L. \& WAXMAN, S. G. 2005. International Union of Pharmacology. XLVII. Nomenclature and structure-function relationships of voltage-gated sodium channels. Pharmacol Rev, 57, 397-409.

CHATTERJEE, A., XIAO, H., BOLLONG, M., Al, H. W. \& SCHULTZ, P. G. 2013. Efficient viral delivery system for unnatural amino acid mutagenesis in mammalian cells. Proc Natl Acad Sci U S A, 110, 118038.

CHIN, J. W. 2017. Expanding and reprogramming the genetic code. Nature, 550, 53-60.

CRANER, M. J., NEWCOMBE, J., BLACK, J. A., HARTLE, C., CUZNER, M. L. \& WAXMAN, S. G. 2004. Molecular changes in neurons in multiple sclerosis: altered axonal expression of Nav1.2 and Nav1.6 sodium channels and $\mathrm{Na}+\mathrm{Ca2}+$ exchanger. Proc Natl Acad Sci U S A, 101, 8168-73.

DUMITRESCU, A. S., EVANS, M. D. \& GRUBB, M. S. 2016. Evaluating Tools for Live Imaging of Structural Plasticity at the Axon Initial Segment. Front Cell Neurosci, 10, 268.

DZHASHIASHVILI, Y., ZHANG, Y., GALINSKA, J., LAM, I., GRUMET, M. \& SALZER, J. L. 2007. Nodes of Ranvier and axon initial segments are ankyrin G-dependent domains that assemble by distinct mechanisms. J Cell Biol, 177, 857-70.

ERNST, R. J., KROGAGER, T. P., MAYWOOD, E. S., ZANCHI, R., BERANEK, V., ELLIOTT, T. S., BARRY, N. P., HASTINGS, M. H. \& CHIN, J. W. 2016. Genetic code expansion in the mouse brain. Nat Chem Biol, 12, 776-778.

EVANS, M. D., DUMITRESCU, A. S., KRUIJSSEN, D. L. H., TAYLOR, S. E. \& GRUBB, M. S. 2015. Rapid Modulation of Axon Initial Segment Length Influences Repetitive Spike Firing. Cell Rep, 13, $1233-$ 1245.

FAKHIRI, J. \& GRIMM, D. 2021. Best of most possible worlds: Hybrid gene therapy vectors based on parvoviruses and heterologous viruses. Mol Ther, 29, 3359-3382.

FELDMAN, D. H. \& LOSSIN, C. 2014. The Nav channel bench series: Plasmid preparation. MethodsX, 1, 611.

FREAL, A., RAI, D., TAS, R. P., PAN, X., KATRUKHA, E. A., VAN DE WILLIGE, D., STUCCHI, R., AHER, A., YANG, C., ALTELAAR, A. F. M., VOCKING, K., POST, J. A., HARTERINK, M., KAPITEIN, L. C., AKHMANOVA, A. \& HOOGENRAAD, C. C. 2019. Feedback-Driven Assembly of the Axon Initial Segment. Neuron, 104, 305-321 e8.

GALIANO, M. R., JHA, S., HO, T. S., ZHANG, C., OGAWA, Y., CHANG, K. J., STANKEWICH, M. C., MOHLER, P. J. \& RASBAND, M. N. 2012. A distal axonal cytoskeleton forms an intra-axonal boundary that controls axon initial segment assembly. Cell, 149, 1125-39.

GASSER, A., HO, T. S., CHENG, X., CHANG, K. J., WAXMAN, S. G., RASBAND, M. N. \& DIB-HAJJ, S. D. 2012. An ankyring-binding motif is necessary and sufficient for targeting Nav1.6 sodium channels to axon initial segments and nodes of Ranvier. $J$ Neurosci, 32, 7232-43.

GHOSH, A., MALAVASI, E. L., SHERMAN, D. L. \& BROPHY, P. J. 2020. Neurofascin and Kv7.3 are delivered to somatic and axon terminal surface membranes en route to the axon initial segment. Elife, 9.

GRIMM, D. \& ZOLOTUKHIN, S. 2015. E Pluribus Unum: 50 Years of Research, Millions of Viruses, and One Goal--Tailored Acceleration of AAV Evolution. Mol Ther, 23, 1819-31. 
1052

1053

1054

1055

1056

1057

1058

1059

1060

1061

1062

1063

1064

1065

1066

1067

1068

1069

1070

1071

1072

1073

1074

1075

1076

1077

1078

1079

1080

1081

1082

1083

1084

1085

1086

1087

1088

1089

1090

1091

1092

1093

1094

1095

1096

1097

1098

1099

GRUBB, M. S. 2021. AIS quantification (https://www.mathworks.com/matlabcentral/fileexchange/28181ais-quantification), MATLAB Central File Exchange. Retrieved December 22, 2021. .

GRUBB, M. S. \& BURRONE, J. 2010. Activity-dependent relocation of the axon initial segment fine-tunes neuronal excitability. Nature, 465, 1070-4.

HAMDAN, H., LIM, B. C., TORII, T., JOSHI, A., KONNING, M., SMITH, C., PALMER, D. J., NG, P., LETERRIER, C., OSES-PRIETO, J. A., BURLINGAME, A. L. \& RASBAND, M. N. 2020. Mapping axon initial segment structure and function by multiplexed proximity biotinylation. Nat Commun, 11, 100.

HEDSTROM, K. L., OGAWA, Y. \& RASBAND, M. N. 2008. Ankyring is required for maintenance of the axon initial segment and neuronal polarity. $J$ Cell Biol, 183, 635-40.

HU, W., TIAN, C., LI, T., YANG, M., HOU, H. \& SHU, Y. 2009. Distinct contributions of $\mathrm{Na(v)1.6}$ and $\mathrm{Na}(\mathrm{v}) 1.2$ in action potential initiation and backpropagation. Nat Neurosci, 12, 996-1002.

HUANG, B., WANG, W., BATES, M. \& ZHUANG, X. 2008. Three-dimensional super-resolution imaging by stochastic optical reconstruction microscopy. Science, 319, 810-3.

JENKINS, P. M., KIM, N., JONES, S. L., TSENG, W. C., SVITKINA, T. M., YIN, H. H. \& BENNETT, V. 2015. Giant ankyrin-G: a critical innovation in vertebrate evolution of fast and integrated neuronal signaling. Proc Natl Acad Sci U S A, 112, 957-64.

JOHANNESEN, K. M., LIU, Y., KOKO, M., GJERULFSEN, C. E., SONNENBERG, L., SCHUBERT, J., FENGER, C. D., ELTOKHI, A., RANNAP, M., KOCH, N. A., LAUXMANN, S., KRUGER, J., KEGELE, J., CANAFOGLIA, L., FRANCESCHETTI, S., MAYER, T., REBSTOCK, J., ZACHER, P., RUF, S., ALBER, M., STERBOVA, K., LASSUTHOVA, P., VLCKOVA, M., LEMKE, J. R., PLATZER, K., KREY, I., HEINE, C., WIECZOREK, D., KROELL-SEGER, J., LUND, C., KLEIN, K. M., BILLIE AU, P. Y., RHO, J. M., HO, A. W., MASNADA, S., VEGGIOTTI, P., GIORDANO, L., ACCORSI, P., HOEI-HANSEN, C. E., STRIANO, P., ZARA, F., VERHELST, H., VERHOEVEN, J. S., VAN DER ZWAAG, B., HARDER, A. V. E., BRILSTRA, E., PENDZIWIAT, M., LEBON, S., VACCAREZZA, M., MINH LE, N., CHRISTENSEN, J., GRONBORG, S., SCHERER, S. W., HOWE, J., FAZELI, W., HOWELL, K. B., LEVENTER, R., STUTTERD, C., WALSH, S., GERARD, M., GERARD, B., MATRICARDI, S., BONARDI, C. M., SARTORI, S., BERGER, A., HOFFMAN-ZACHARSKA, D., MASTRANGELO, M., DARRA, F., VOLLO, A., MOTAZACKER, M. M., LAKEMAN, P., NIZON, M., BetZleR, C., ALTUZARRA, C., CAUME, R., ROUBERTIE, A., GEliSSE, P., MARINI, C., GUERRINI, R., BILAN, F., TIBUSSEK, D., KOCH-HOGREBE, M., PERRY, M. S., ICHIKAWA, S., DADALI, E., SHARKOV, A., MISHINA, I., ABRAMOV, M., KANIVETS, I., KOROSTELEV, S., KUTSEV, S., WAIN, K. E., EISENHAUER, N., WAGNER, M., SAVATT, J. M., MULLER-SCHLUTER, K., BASSAN, H., BOROVIKOV, A., NASSOGNE, M. C., DESTREE, A., et al. 2021. Genotype-phenotype correlations in SCN8Arelated disorders reveal prognostic and therapeutic implications. Brain.

KIM, J., ADAM, R. M., SOLOMON, K. R. \& FREEMAN, M. R. 2004. Involvement of cholesterol-rich lipid rafts in interleukin-6-induced neuroendocrine differentiation of LNCaP prostate cancer cells. Endocrinology, 145, 613-9.

KIRA, J. I., YAMASAKI, R. \& OGATA, H. 2019. Anti-neurofascin autoantibody and demyelination. Neurochem Int, 130, 104360.

KOLE, M. H., ILSCHNER, S. U., KAMPA, B. M., WILLIAMS, S. R., RUBEN, P. C. \& STUART, G. J. 2008. Action potential generation requires a high sodium channel density in the axon initial segment. Nat Neurosci, 11, 178-86.

LANG, K., DAVIS, L., TORRES-KOLBUS, J., CHOU, C., DEITERS, A. \& CHIN, J. W. 2012a. Genetically encoded norbornene directs site-specific cellular protein labelling via a rapid bioorthogonal reaction. Nat Chem, 4, 298-304.

LANG, K., DAVIS, L., WALLACE, S., MAHESH, M., COX, D. J., BLACKMAN, M. L., FOX, J. M. \& CHIN, J. W. 2012b. Genetic Encoding of bicyclononynes and trans-cyclooctenes for site-specific protein labeling in vitro and in live mammalian cells via rapid fluorogenic Diels-Alder reactions. J Am Chem Soc, 134, 10317-20. 
LEFFLER, A., HERZOG, R. I., DIB-HAJJ, S. D., WAXMAN, S. G. \& CUMMINS, T. R. 2005. Pharmacological properties of neuronal TTX-resistant sodium channels and the role of a critical serine pore residue. Pflugers Arch, 451, 454-63.

LETERRIER, C. 2018. The Axon Initial Segment: An Updated Viewpoint. J Neurosci, 38, 2135-2145.

LETERRIER, C., POTIER, J., CAILLOL, G., DEBARNOT, C., RUEDA BORONI, F. \& DARGENT, B. 2015. Nanoscale Architecture of the Axon Initial Segment Reveals an Organized and Robust Scaffold. Cell Rep, 13, 2781-93.

LIU, H., WANG, H.-G., PITT, G. S. \& LIU, Z. J. 2021. Nanometer-Scale Imaging of Compartment-Specific Localization and Dynamics of Voltage-Gated Sodium Channels. bioRxiv, 2021.09.28.462114.

LIU, Y., SCHUBERT, J., SONNENBERG, L., HELBIG, K. L., HOEI-HANSEN, C. E., KOKO, M., RANNAP, M., LAUXMANN, S., HUQ, M., SCHNEIDER, M. C., JOHANNESEN, K. M., KURLEMANN, G., GARDELLA, E., BECKER, F., WEBER, Y. G., BENDA, J., MOLLER, R. S. \& LERCHE, H. 2019. Neuronal mechanisms of mutations in SCN8A causing epilepsy or intellectual disability. Brain, 142, 376-390.

MEIJERING, E., JACOB, M., SARRIA, J. C., STEINER, P., HIRLING, H. \& UNSER, M. 2004. Design and validation of a tool for neurite tracing and analysis in fluorescence microscopy images. Cytometry $A, 58,167-$ 76.

MEISLER, M. H., HILL, S. F. \& YU, W. 2021. Sodium channelopathies in neurodevelopmental disorders. Nat Rev Neurosci, 22, 152-166.

NIKIC-SPIEGEL, I. 2020. Expanding the Genetic Code for Neuronal Studies. Chembiochem, 21, 3169-3179.

NIKIC, I., PLASS, T., SCHRAIDT, O., SZYMANSKI, J., BRIGGS, J. A., SCHULTZ, C. \& LEMKE, E. A. 2014. Minimal tags for rapid dual-color live-cell labeling and super-resolution microscopy. Angew Chem Int Ed Engl, 53, 2245-9.

O'BRIEN, J. E. \& MEISLER, M. H. 2013. Sodium channel SCN8A (Nav1.6): properties and de novo mutations in epileptic encephalopathy and intellectual disability. Front Genet, 4, 213.

PLASS, T., MILLES, S., KOEHLER, C., SZYMANSKI, J., MUELLER, R., WIESSLER, M., SCHULTZ, C. \& LEMKE, E. A. 2012. Amino acids for Diels-Alder reactions in living cells. Angew Chem Int Ed Engl, 51, 416670.

SCHAFER, D. P., JHA, S., LIU, F., AKELLA, T., MCCULLOUGH, L. D. \& RASBAND, M. N. 2009. Disruption of the axon initial segment cytoskeleton is a new mechanism for neuronal injury. J Neurosci, 29, 1324254.

SCHINDELIN, J., ARGANDA-CARRERAS, I., FRISE, E., KAYNIG, V., LONGAIR, M., PIETZSCH, T., PREIBISCH, S., RUEDEN, C., SAALFELD, S., SCHMID, B., TINEVEZ, J. Y., WHITE, D. J., HARTENSTEIN, V., ELICEIRI, K., TOMANCAK, P. \& CARDONA, A. 2012. Fiji: an open-source platform for biological-image analysis. Nat Methods, 9, 676-82.

SCHMIED, W. H., ELSASSER, S. J., UTTAMAPINANT, C. \& CHIN, J. W. 2014. Efficient multisite unnatural amino acid incorporation in mammalian cells via optimized pyrrolysyl tRNA synthetase/tRNA expression and engineered eRF1. J Am Chem Soc, 136, 15577-83.

SHARKEY, L. M., CHENG, X., DREWS, V., BUCHNER, D. A., JONES, J. M., JUSTICE, M. J., WAXMAN, S. G., DIBHAJJ, S. D. \& MEISLER, M. H. 2009. The ataxia3 mutation in the N-terminal cytoplasmic domain of sodium channel $\mathrm{Na}(\mathrm{v}) 1.6$ disrupts intracellular trafficking. J Neurosci, 29, 2733-41.

SIZOVA, D. V., HUANG, J., AKIN, E. J., ESTACION, M., GOMIS-PEREZ, C., WAXMAN, S. G. \& DIB-HAJJ, S. D. 2020. A 49-residue sequence motif in the $C$ terminus of Nav1.9 regulates trafficking of the channel to the plasma membrane. J Biol Chem, 295, 1077-1090.

SOLE, L. \& TAMKUN, M. M. 2020. Trafficking mechanisms underlying Nav channel subcellular localization in neurons. Channels (Austin), 14, 1-17.

SOLE, L., WAGNON, J. L., AKIN, E. J., MEISLER, M. H. \& TAMKUN, M. M. 2019. The MAP1B Binding Domain of Nav1.6 Is Required for Stable Expression at the Axon Initial Segment. J Neurosci, 39, 4238-4251. 
SUSUKI, K., CHANG, K. J., ZOLLINGER, D. R., LIU, Y., OGAWA, Y., ESHED-EISENBACH, Y., DOURSZIMMERMANN, M. T., OSES-PRIETO, J. A., BURLINGAME, A. L., SEIDENBECHER, C. I., ZIMMERMANN, D. R., OOHASHI, T., PELES, E. \& RASBAND, M. N. 2013. Three mechanisms assemble central nervous system nodes of Ranvier. Neuron, 78, 469-82.

TORII, T., OGAWA, Y., LIU, C. H., HO, T. S., HAMDAN, H., WANG, C. C., OSES-PRIETO, J. A., BURLINGAME, A. L. \& RASBAND, M. N. 2020. NuMA1 promotes axon initial segment assembly through inhibition of endocytosis. J Cell Biol, 219.

UTTAMAPINANT, C., HOWE, J. D., LANG, K., BERANEK, V., DAVIS, L., MAHESH, M., BARRY, N. P. \& CHIN, J. W. 2015. Genetic code expansion enables live-cell and super-resolution imaging of site-specifically labeled cellular proteins. J Am Chem Soc, 137, 4602-5.

VON CASTELMUR, E., MARINO, M., SVERGUN, D. I., KREPLAK, L., UCURUM-FOTIADIS, Z., KONAREV, P. V., URZHUMTSEV, A., LABEIT, D., LABEIT, S. \& MAYANS, O. 2008. A regular pattern of Ig super-motifs defines segmental flexibility as the elastic mechanism of the titin chain. Proc Natl Acad Sci U SA, $105,1186-91$.

WANG, L. 2017. Engineering the Genetic Code in Cells and Animals: Biological Considerations and Impacts. Acc Chem Res, 50, 2767-2775.

XU, K., ZHONG, G. \& ZHUANG, X. 2013. Actin, spectrin, and associated proteins form a periodic cytoskeletal structure in axons. Science, 339, 452-6.

ZHANG, X., DAVIS, J. Q., CARPENTER, S. \& BENNETT, V. 1998. Structural requirements for association of neurofascin with ankyrin. J Biol Chem, 273, 30785-94.

ZHENG, Y., LEWIS, T. L., JR., IGO, P., POLLEUX, F. \& CHATTERJEE, A. 2017. Virus-Enabled Optimization and Delivery of the Genetic Machinery for Efficient Unnatural Amino Acid Mutagenesis in Mammalian Cells and Tissues. ACS Synth Biol, 6, 13-18. 